\title{
NOTE ON THE FILTRATIONS OF THE $K$-THEORY
}

\author{
NOBUAKI YAGITA
}

\begin{abstract}
Let $X$ be a (colimit of) smooth algebraic variety over a subfield of $\mathbb{C}$. Let $K_{\text {alg }}^{0}(X)$ (resp. $K_{\text {top }}^{0}(X(\mathbb{C}))$ ) be the algebraic (resp. topological) $K$-theory of $k$ (resp. complex) vector bundles over $X(\operatorname{resp} . X(\mathbb{C}))$ ). When $K_{\text {alg }}^{0}(X) \cong K_{\text {top }}^{0}(X(\mathbb{C}))$, we study the differences of its three (gamma, geometrical and topological) filtrations. In particular, we consider the cases $X=B G$ for an algebraically closed field $k$, and $X=\mathbb{G}_{k} / T_{k}$ the twisted form of flag varieties $G / T$ for non-algebraically closed field $k$.
\end{abstract}

\section{INTRODUCTION}

Let $X$ be a (colimit of) smooth algebraic variety over a subfield $k$ of $\mathbb{C}$. We consider the cases that

$$
\text { (1.1) } \quad K_{\text {alg }}^{0}(X) \cong K_{\text {top }}^{0}(X(\mathbb{C}))
$$

where $K_{\text {alg }}^{0}(X)\left(\right.$ resp. $\left.K_{\text {top }}^{0}(X(\mathbb{C}))\right)$ is the algebraic (resp. topological) $K$-theory generated by algebraic $k$-bundles (complex bundles) over $X$ (resp. $X(\mathbb{C})$ ). In this assumption, we study the typical three filtrations

$$
F_{\gamma}^{i}(X) \subset F_{\text {geo }}^{i}(X) \subset F_{\text {top }}^{i}(X(\mathbb{C}))
$$

namely, the gamma and the geometric filtrations defined by Grothendieck $[\mathrm{Gr}]$, and the topological filtration defined by Atiyah [At]. Namely, we study induced maps of associated rings

$$
g r_{\gamma}^{*}(X) \rightarrow g r_{\text {geo }}^{*}(X) \rightarrow g r_{\text {top }}^{*}(X(\mathbb{C})) .
$$

Atiyah showed that $g r_{\text {top }}^{*}(X(\mathbb{C}))$ is isomorphic to the infitite term $E_{\infty}^{*, 0}$ of the AHss (Atiyah-Hirzebruch spectral sequence) converging to $K$-theory $K^{*}(X(\mathbb{C})$ ). Moreover he showed that $g r_{\text {top }}^{*}(X(\mathbb{C})) \cong g r_{\gamma}^{*}(X)$ if and only if $E_{\infty}^{*, 0}$ is generated by Chern classes in $H^{*}(X(\mathbb{C}))$. We will see that similar facts hold for $g r_{g e o}^{*}(X)$. Namely, $g r_{g e o}^{2 *}(X) \cong A E_{\infty}^{2 *, * 0}$ of the motivic AHss converging to motivic $K$-theory $A K^{*, *^{\prime}}(X)$. Moreover we show that $g r_{g e o}^{*}(X) \cong g r_{\gamma}^{*}(X)$ if and only if $A E_{\infty}^{2 *, *, 0}$ is generated by Chern classes in the Chow ring $C H^{*}(X) \cong H^{2 *, *}(X)$.

Let $G$ be a compact Lie group (e.g., a finite group) and $G_{k}$ be the corresponding algebraic group over an algebraically closed field $k$. Then by Merkurjev and Totaro $([\mathrm{To}])$, we have the isomorphisms

$$
K_{\text {alg }}^{0}\left(B G_{k}\right) \cong R\left(G_{k}\right)^{\wedge} \cong R(G)^{\wedge} \cong K_{\text {top }}^{0}(B G)
$$

where $R\left(G_{k}\right)^{\wedge}$ (resp. $R(G)^{\wedge}$ ) is the $k$-representation (resp. complex representation) ring completed by the augmentation ideal, and $B G_{k}$ and $B G$ are their classifying spaces.

2000 Mathematics Subject Classification. Primary 11E72, Secondary 12G05.

Key words and phrases. $\gamma$-filtration, K-theory, representation ring. 
Atiyah had conjectured in $[\mathrm{At}]$ that $F_{\gamma}^{i}(B G)=F_{\text {top }}^{i}(B G)$ for all finite groups. Weiss [Th] showed this does not hold for $G=A_{4}$. For counter examples of $p$-groups were given by Leary-Yagita [Le-Ya] when $G$ is $\operatorname{rank}_{p}(G)=2$ of class 3 with $p \geq 5$. We will see for the same group $G, F_{\gamma}^{2 p+2}\left(B G_{k}\right) \neq F_{g e o}^{2 p+2}\left(B G_{k}\right)=F_{t o p}^{2 p+2}\left(B G_{k}\right)$.

We study these filtrations detailedly for connected groups $\left(O_{n}, S O_{n}, \ldots\right)$. In particular we show

Theorem 1.1. (Let $k$ be an algebraically closed field.) For $G=\operatorname{Spin}_{7}$, there is an element $x$ in $K_{\text {alg }}^{0}\left(B G_{k}\right)$ such that

$$
0 \neq x \in g r_{\gamma}^{4}\left(B G_{k}\right), \quad 0 \neq x \in g r_{g e o}^{6}\left(B G_{k}\right), \quad 0 \neq x \in g r_{\text {top }}^{8}(B G) .
$$

These facts also hold for the extraspecial 2-group $2_{+}^{1+6}$.

We consider the different type of examples, which satisfy (1.1). (See also [Ga$\mathrm{Za}],[\mathrm{Za}]$.) Here we do not assume that $k$ is algebraically closed. Let us write by $M(X)$ the (pure) motive of $X$, and by $M_{a}=\left(M_{n}\right)$ the Rost motive for a nonzero pure symbol $a \in K_{n+1}^{M}(k) / p$ ([Ro1,2], [Su-Jo]). We consider the cases $X$ such that

$$
\text { (1.2) } M(X) \cong M_{n} \otimes A(X)
$$

where $A(X)$ is a sum of $k$-Tate motives. Then we can see that (1.1) is satisfied by the result from([Vi-Ya],[Ya]).

Some cases of flag manifolds $G / P$ satisfy (1.2) ([Ca-Pe-Se-Za], [Ni-Se-Za], [PeSe-Za]). In particular (for $p=2) X=\mathbb{G}_{2, k} / T_{k}$ is a such example, where $\mathbb{G}_{2, k}$ is the nontrivial $G_{2, k}$-torsor (induced from a Rost cohomological invariant $0 \neq a \in$ $K_{3}^{M}(k) / 2$, [Ga-Me-Se]) for the exceptional Lie group $G_{2}$, and $T$ a maximal torus in $G$ ([Bo], [Pe-Se-Za]). (Namely, $\mathbb{G}_{2, k} / T_{k}$ is a twisted form of $G_{2} / T$.) Note that $H^{*}\left(G_{2} / T\right)$ is torsion free, and we have

$$
g r_{\text {geo }}^{*}\left(G_{2, k} / T_{k}\right) \cong g r_{\text {top }}^{*}\left(G_{2} / T\right) \cong H^{*}\left(G_{2} / T\right) .
$$

By using the fact that $C H^{*}\left(\mathbb{G}_{2, k} / T_{k}\right)$ is generated by Chern classes, we can show

Theorem 1.2. Let $\mathbb{G}_{2, k}$ be the nontrivial $G_{2, k}$-torsor for the Rost cohomological invariant in $K_{3}^{M}(k) / 2$. Then we have

$$
g r_{\gamma}^{2 *}\left(G_{2} / T\right) \cong g r_{g e o}^{2 *}\left(\mathbb{G}_{2, k} / T_{k}\right) \cong C H^{*}\left(\mathbb{G}_{2, k} / T_{k}\right) .
$$

From (1.1), the gamma filtration is defined purely topologically. Thus we see that this topological invariant is isomorphic to a purely algebraic geometric object such as the Chow ring of twisted form.

\section{FILTRATIONS}

We first recall the topological filtration defined by Atiyah. Let $Y$ be a topological space (e.g., finitely generated $C W$-complex). Let $K^{*}(Y)$ be the complex $K$-theory ; the Grothendieck group generated by complex bundles over $Y$. Let $Y^{i}$ be an $i$-dimensional skeleton of $Y$. Define the topological filtration of $K^{*}(Y)$ by

$$
F_{\text {top }}^{i}(Y)=\operatorname{Ker}\left(K^{*}(Y) \rightarrow K^{*}\left(Y^{i}\right)\right)
$$

and the associated graded algebra $g r_{\text {top }}^{i}(Y)=F_{\text {top }}^{i}(Y) / F_{\text {top }}^{i+1}(Y)$.

We consider the long exact sequence (exact couple)

$$
\ldots \rightarrow K^{*}\left(Y^{i} / Y^{i-1}\right) \rightarrow K^{*}\left(Y^{i}\right) \rightarrow K^{*}\left(Y^{i-1}\right) \stackrel{\delta}{\rightarrow} K^{*+1}\left(Y^{i} / Y^{i-1}\right) \rightarrow \ldots
$$


Here we have $K^{*}\left(Y^{i} / Y^{i-1}\right) \cong K^{*} \otimes H^{*}\left(Y^{i} / Y^{i-1}\right)$, which induces the (well known) AHss

$$
E_{2}^{*, *^{\prime}}(Y) \cong H^{*}(Y) \otimes K^{*} \Longrightarrow K^{*}(Y) .
$$

By the construction of the spectral sequence, we have

Lemma 2.1. (Atiyah $[$ At] $) g r_{\text {top }}^{*}(Y) \cong E_{\infty}^{*, 0}(Y)$.

Next we consider the geometric filtration. Let $X$ be a smooth algebraic variety over a subfield $k$ of $\mathbb{C}$. Let $K_{\text {alg }}^{0}(X)$ be the algebraic $K$-theory which is the Grothendiek group generated by $k$-vector bundles over $X$. It is also isomorphic to the Grothendieck group genrated by coherent sheaves over $X$ (we assumed $X$ smooth). This $K$-theory can be written by the motivic $K$-theory $A K^{*, *^{\prime}}(Y)$ ([Vo1,2], i.e.,

$$
K_{\text {alg }}^{i}(X)=\oplus_{*} A K^{2 *-i, *}(X) .
$$

In particular $K_{\text {alg }}^{0}(X)=\oplus_{*} A K^{2 *, *}(X)$.

The geometric filtration $([\mathrm{Gr}])$ is defined as

$$
F_{\text {geo }}^{i}(X)=\left\{\left[O_{V}\right] \mid \operatorname{codim}_{X} V \geq i\right\}
$$

(and $\left.F_{\text {geo }}^{2 i-1}(X)=F_{\text {geo }}^{2 i}(X)\right)$ where $O_{V}$ is the structural sheaf of closed subvariety $V$ of $X$.

We recall the algebraic cobordism $M G L^{*, *^{\prime}}(-)$ [Vo1] and let us write $M G L^{2 *, *}(X)=$ $\Omega^{*}(X)$, in fact, this is isomorphic to the algebraic cobordism defined by Levine and Morel ([Le-Mo1,2], [Vo1,2]). Recall

$$
\Omega^{*}(\operatorname{Spec}(k))=\Omega^{*}(p t .) \cong M U^{2 *}(p t .)=M U^{*} .
$$

Then we have the isomorphism

$$
\Omega^{*}(X) \otimes_{M U^{*}} \mathbb{Z} \cong C H^{*}(X), \quad \Omega^{*}(X) \otimes_{M U^{*}} K^{*} \cong K_{\text {alg }}^{0}(X)
$$

where the $M U^{*}$ module structure of $K^{*}$ is given by Todd genus (see $\S$ below). Each element $x \in \Omega^{*}(X)$ is represented by a projective map $x=[f: M \rightarrow X]$ with $\operatorname{codim}_{X} M=i$ and $M$ smooth ([Le-Mo1,2]), namely, $x=f_{*}\left(1_{M}\right)$ for $1_{M} \in \Omega^{0}(M)$ and $f_{*}$ is the Gysin map. Then the geometric filtration is also defined as

$$
F_{\text {geo }}^{2 i}(X)=\left\{f_{*}\left(1_{M}\right) \mid f: M \rightarrow X \text { and } \operatorname{codim}_{X} M \geq i\right\} .
$$

Here we recall the motivic AHss ([Ya2, 4])

$$
A E_{2}^{*, *^{\prime}, *^{\prime \prime}}(X) \cong H^{*, *^{\prime}}\left(X ; K^{*^{\prime \prime}}\right) \Longrightarrow A K^{*, *^{\prime}}(X) .
$$

(Of course this spectral sequence is not defined using skeleton as the topological case. But we assume the existence of the AHss converging to the motivic $K$-theory $A K^{*, *^{\prime}}(X)$.) Note that

$$
A E_{2}^{2 *, *, *^{\prime \prime}}(X) \cong H^{2 *, *}\left(X ; K^{*^{\prime \prime}}\right) \cong C H^{*}(X) \otimes K^{*^{\prime \prime}} .
$$

Hence $A E_{\infty}^{2 *, *, 0}(X)$ is a quotient of $C H^{*}(X)$ by dimensional reason of degree of differential $d_{r}$ (i.e., $d_{r} A E_{r}^{2 * * * *^{\prime \prime}}(X)=0$ ). Thus we have

Lemma 2.2. $g r_{g e o}^{2 *}(X) \cong A E_{\infty}^{2 *, *, 0}(X)$. 
Proof. Let $q: \Omega^{*}(X) \otimes K^{*} \rightarrow K^{*}(X)$. Then

$$
F_{g e o}^{2 i}(X)=q\left\{f_{*}\left(1_{M}\right) \in \Omega^{*}(X) \mid f: M \rightarrow X \text { and } \operatorname{codim}_{X} M \geq i\right\} .
$$

Let $q^{\prime}: \Omega^{*}(X) \rightarrow C H^{*}(X)$ and $q^{\prime \prime}: C H^{*}(X) \rightarrow E_{\infty}^{2 *, *, 0}$. Then $q \mid\left(\Omega^{*}(X) \otimes 1\right)=$ $q^{\prime \prime} q^{\prime}$. Thus we have

$$
F_{g e o}^{2 i}(X) / F_{g e o}^{2 i+2}(X)=q^{\prime \prime} C H^{i}(X)
$$

since $q^{\prime}$ is an epimorphism.

Lemma 2.3. Let $t_{\mathbb{C}}: K_{\text {alg }}^{0}(X) \rightarrow K_{\text {top }}^{0}(X(\mathbb{C}))$ be the realization map. Then $F_{\text {geo }}^{i}(X) \subset\left(t_{\mathbb{C}}^{*}\right)^{-1} F_{\text {top }}^{i}(X(\mathbb{C}))$.

Proof. Let us write $K_{\text {top }}^{0}(X(\mathbb{C}))$ simply by $K(X)$. The Gysin map $f_{*}: K(M) \rightarrow$ $K(X)$ is defined by using Thom isomorphism

$$
K(M) \cong K\left(T h_{X}(M)\right) \rightarrow K(X) .
$$

Let $\operatorname{codim}_{X} M \geq i$. For an $2 i$-skeleton $X^{2 i}$ of $X(\mathbb{C})$, we can show that the map

$$
K\left(T h_{X}(M)\right) \rightarrow K(X) \rightarrow K\left(X^{2 i}\right)
$$

is zero. Because the above composition map is rewritten

$$
K\left(T h_{X}(M)\right) \rightarrow K\left(T h_{X}(M)^{2 i}\right) \rightarrow K\left(X^{2 i}\right) .
$$

Its first map is zero, because $H^{*}\left(T h_{X}(M)\right)=0$ for $*<2 i$ and the exact sequence (exact couple) for $K$-theory for skeletons of $X$ (see the definition of the AHss).

At last, we consider the gamma filtration. Let $\lambda^{i}(x)$ be the exterior power of the vector bundle $x \in K_{\text {alg }}^{0}(X)$ and $\lambda_{t}(x)=\sum \lambda^{i}(x) t^{i}$. Let us denote

$$
\lambda_{t /(1-t)}(x)=\gamma_{i}(x)=\sum \gamma^{i}(x) t^{i}
$$

The Gamma filtration is defined as

$$
F_{\gamma}^{2 i}(X)=\left\{\gamma^{i_{1}}\left(x_{1}\right) \cdot \ldots \cdot \gamma^{i_{m}}\left(x_{m}\right) \mid i_{1}+\ldots+i_{m} \geq i, x_{j} \in K_{\text {alg }}^{0}(X)\right\}
$$

Then we can see $F_{\gamma}^{i}(X) \subset F_{g e o}^{i}(X)$ (Proposition 12.5 in [At], Atiyah proved $F_{\gamma}^{i}(X) \subset F_{\text {top }}^{i}(X)$ in $K_{\text {top }}(X)$. However the arguments work also in $K_{\text {alg }}^{0}(X)$ and this fact is well known.) Let $\epsilon: K_{\text {alg }}^{0}(X) \rightarrow \mathbb{Z}$ be the augmentation map and $c_{i}(x) \in H^{2 i, i}(X)$ the Chern class. Recall $q^{\prime \prime}: C H^{*}(X) \rightarrow E_{\infty}^{2 *, *, 0}$ be the quotient map. Then (p. 63 in $[\mathrm{At}])$ we have

$$
q^{\prime \prime}\left(c_{n}(x)\right)=\left[\gamma^{n}(x-\epsilon(x))\right] .
$$

Lemma 2.4. (Atiyah) The condition $F_{\gamma}^{2 *}(Y)=F_{\text {top }}^{2 *}(Y)\left(\operatorname{resp} . F_{\gamma}^{2 *}(X)=F_{\text {geo }}^{2 *}(X)\right)$ is equivalent to that $E_{\infty}^{2 *, 0}(Y)$ (resp. $\left.A E_{\infty}^{2 *, *, 0}(X)\right)$ is (multiplicatively) generated by Chern classes in $H^{2 *}(B G)$ (resp. $\left.C H^{*}(B G)\right)$. 


\section{Morava $K$-theory ( $K$-THeory LOCALized at $p$ )}

In this paper, we assume that $p$ is a fixed prime number and consider only cohomology theories (Chern rings) localized at this prime $p$. Namely, for the notation $A^{*}(X)$ means $A^{*}(X)_{(p)}$ in this paper. In particular, $\mathbb{Z}$ always means $\mathbb{Z}_{(p)}$ and $M U^{*}(-)$ means $M U^{*}(X)_{(p)}$ throughout this paper.

Let $A M U^{*, *^{\prime}}(X)=M G L^{*, *^{\prime}}(X)$ and recall $M U^{*}=\mathbb{Z}\left[x_{1}, \ldots, x_{n}, ..\right], \operatorname{deg}\left(x_{i}\right)=$ $(-2 i,-i)$. Given a sequence $S=\left(x_{i_{1}}, x_{i_{2}}, \ldots\right)$ of generators, we can construct generalized cohomology theory (in the $\mathbb{A}^{1}$-homotopy category) such that

$$
t_{\mathbb{C}} A M U(S)^{*, *^{\prime}}(X)=M U(S)^{*}(X(\mathbb{C})) \text { with } M U(S)^{*}=M U^{*} /(S) .
$$

In particular letting $x_{p^{n}-1}=v_{n}$ and $S=\left(x_{i} \mid i \neq p^{n}-1\right)$, we have the motivic $B P$-theory $([\mathrm{Ya} 2,4])$

$$
A B P^{*, *^{\prime}}(X) \text { with } M U^{*} /(S) \cong B P^{*}=\mathbb{Z}\left[v_{1}, v_{2}, \ldots\right] .
$$

Then we have the isomorphisms ([Ya])

$$
\begin{aligned}
& A B P^{*, *^{\prime}}(X) \cong M G L^{*, *^{\prime}}(X) \otimes_{M U^{*}} B P^{*}, \\
& M G L^{*, *^{\prime}}(X) \cong A B P^{*, *^{\prime}}(X) \otimes_{B P^{*}} M U^{*} .
\end{aligned}
$$

Similarly, we can construct the motivic connective Morava $K$-theory such that

$$
A k(1)^{*, *^{\prime}}(X) \text { with } k(1)^{*}=\mathbb{Z} / p\left[v_{n}\right],
$$

and the integral connected $K$-theory $A \tilde{k}(1)^{*, *^{\prime}}(X)$ with $\tilde{k}(n)=\mathbb{Z}\left[v_{n}\right]$. Moreover let the (usual) motivic Morava $K$-theory

$$
A K(n)^{*, *^{\prime}}(X)=A k(n)^{*, *^{\prime}}(X)\left[v_{n}^{-1}\right], \quad A \tilde{K}(n)^{*, *^{\prime}}(X)=A \tilde{k}(n)^{*, *^{\prime}}(X)\left[v_{n}^{-1}\right] .
$$

By the Landweber exact functor theorem ([Ra], [Ha]), it is well known that

$$
A K^{*, *^{\prime}}(X) \cong\left(A M U^{*, *^{\prime}}(X) \otimes_{M U^{*}} \mathbb{Z}\right) \otimes \mathbb{Z}\left[B, B^{-1}\right]
$$

where the $M U^{*}$-module structure of $\mathbb{Z}$ is given by the Todd genus, and $B$ is the Bott periodicity with $\operatorname{deg}(B)=(-2,-1)$. Since the Todd genus of $v_{1}$ (resp. $v_{i}$, $i>1$ ) is 1 (resp. 0 ), we can write

$$
A K^{*, *^{\prime}}(X) \cong A B P^{*, *^{\prime}}(X) \otimes_{B P^{*}} \mathbb{Z}\left[B, B^{-1}\right] \quad \text { identifying } B^{p-1}=v_{1} .
$$

Then we have

Lemma 3.1. There is a natural isomorphism

$$
A \tilde{K}^{*, *^{\prime}}(X) \cong A \tilde{K}(1)^{*, *^{\prime}}(X) \otimes_{\tilde{K}(1)^{*}} \mathbb{Z}\left[B, B^{-1}\right] \quad \text { identifying } v_{1}=B^{p-1} .
$$

Proof. Recall that there is the natural map (by the construction of $A M U(S)$ )

$$
\rho: A B P^{*, *^{\prime}}(X) \otimes_{B P^{*}} \mathbb{Z}\left[B, B^{-1}\right] \rightarrow A \tilde{K}(1)^{*, *^{\prime}}(X) \otimes_{\tilde{K}(1)^{*}} \mathbb{Z}\left[B, B^{-1}\right] .
$$

Of course, the functor

$$
A \mapsto A \otimes_{\tilde{K}(1)^{*}} \mathbb{Z}\left[B, B^{-1}\right] \cong A \otimes \mathbb{Z}\left\{1, B, \ldots, B^{p-2}\right\}
$$

is exact, and we have the spectral sequence

$$
E_{2}^{*, *^{\prime}, *^{\prime \prime}}(A \tilde{K}(1)) \otimes_{\tilde{K}(1)^{*}} \mathbb{Z}\left[B, B^{-1}\right] \Longrightarrow A \tilde{K}(1)^{*, *^{\prime}}(X) \otimes_{\tilde{K}(1)^{*}} \mathbb{Z}\left[B, B^{-1}\right] .
$$

Since for a $B P^{*}(B P)$ module $A$, the functor

$$
A \mapsto A \otimes_{B P^{*}} \mathbb{Z}\left[B, B^{-1}\right]
$$


is exact from the Landweber exact functor theorem, we have the spectral sequence from the AHss for $A B P^{*, *^{\prime}}(X)$

$$
E_{2}^{*, *^{\prime}, *^{\prime \prime}}(A B P) \otimes_{B P^{*}} \mathbb{Z}\left[B, B^{-1}\right] \Longrightarrow A B P^{*, *^{\prime}}(X) \otimes_{B P^{*}} \mathbb{Z}\left[B, B^{-1}\right],
$$

which is compatible with the map $\rho$. The $E_{2}$-term of the both spectral sequences are isomorphic to

$$
H^{*, *^{\prime}}(X ; \mathbb{Z}) \otimes \mathbb{Z}\left[B, B^{-1}\right] .
$$

Therefore the two spectral sequences are isomorphic.

We also note from the arguments in the above proof.

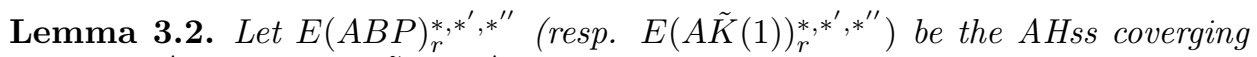
to $A B P^{*, *^{\prime}}(X)$ (resp. $\left.A \tilde{K}(1)^{*, *^{\prime}}(X)\right)$. Then we have

$$
\left.E(A B P)_{r}^{*, *^{\prime}, *^{\prime \prime}} \otimes_{B P^{*}} \tilde{K}(1)^{*} \cong E(A \tilde{K}(1))_{r}^{*, *^{\prime}, *^{\prime \prime}}\right) .
$$

From above lemma, it is sufficient to consider the Morava $K$-theory $A \tilde{K}(1)^{*, *^{\prime}}(X)$ when we want to study $A K^{*, *^{\prime}}(X)$. Hereafter of this paper, we only consider the theories $A \tilde{K}(1)^{*, *^{\prime}}(X)$ and $A \tilde{k}(1)^{*, *^{\prime}}(X)$ instead of $A K^{*, *^{\prime}}(X)$ or $K_{\text {alg }}^{*}(X)$. (We only consider the cohomology theories and Chow rings localied at $p$.)

We assume the following assumption

$$
(*) \quad K_{\text {alg }}^{0}(X) \cong K_{\text {top }}^{0}(X(\mathbb{C})) \quad\left(\text { and } K_{\text {top }}^{1}(X(\mathbb{C}))=0\right) .
$$

That is equivalent to

$$
(*) \quad A \tilde{K}(1)^{2 *, *}(X) \cong \tilde{K}(1)^{2 *}(X(\mathbb{C})) \quad\left(\text { and } \tilde{K}(1)^{2 *+1}(X(\mathbb{C}))=0\right) .
$$

From Lemma 2.3, we have

$$
F_{\gamma}(X) \subset F_{\text {geo }}^{i}(X) \subset F_{\text {top }}^{i}(X(\mathbb{C})) .
$$

Here we note that the gamma filtrations of topogical and algebraic geometrical are same, i.e., $F_{\gamma}^{*}(X) \cong F_{\gamma}^{*}(X(\mathbb{C}))$. So we have the maps of associated graded rings

$$
g r_{\gamma}^{*}(X) \rightarrow g r_{g e o}^{*}(X) \rightarrow g r_{\text {top }}^{*}(X(\mathbb{C})) .
$$

Lemma 3.3. $g r_{\gamma}^{2}(X)=g r_{g e o}^{2}(X)$.

Proof. If $0 \neq x \in g r_{\gamma}^{2}(X)$, then $x_{1}=c_{1}(\xi) \in A \tilde{K}(1)^{2 *, *}(X)$ for some bundle $\xi$. In $C H^{*}(X)$, we know $c_{1}(\xi)=c_{1}(\operatorname{det}(\xi))$ which is determined by the line bundle $\operatorname{det}(\xi)$. Line bundles are determined by $\operatorname{Pic}(X)=C H^{1}(X)$. So $0 \neq x \in C H^{1}(X)$.

Lemma 3.4. If an element $y \in A \tilde{K}(1)^{2 *, *}(X)$ is represented by $0 \neq y^{\prime}$ (resp. $\left.y^{\prime \prime}, y^{\prime \prime \prime}\right) \in$ $g r_{\gamma}^{i}(X)\left(\right.$ resp. $\left.g r_{\text {geo }}^{j}(X), g r_{\text {top }}^{k}(X(\mathbb{C}))\right)$, then

$$
i \leq j \leq k, \quad \text { and } \quad i=k=j \bmod (2(p-1)) .
$$

Proof. The element $y$ is represented

$$
y=v_{1}^{s} y^{\prime} \in A \tilde{K}(1)^{2 *, *}(X) / F_{\gamma}^{2 i+1} \quad y=v_{1}^{t} y^{\prime \prime} \in A \tilde{K}(2)^{2 *, *}(X) / F_{\text {geo }}^{2 j+1}
$$

for some $s, t \in \mathbb{Z} / p$. 
Remark. The above fact does not hold for $y \in K_{\text {top }}^{0}(X)$ (which is a sum of $\left.\tilde{K}(1)^{2 *, *}(X), 0 \leq * \leq p-2\right)$. Let us write

$$
y=B^{k} y_{k}+B^{k+1} y_{k+1}+\ldots+B^{k+p-2} y_{k+p-2},
$$

with $y_{i} \in \tilde{K}(1)^{2 i}(Y)$ and, $y_{i} \in F_{\text {top }}^{2 i}(Y)$. Suppose $j>k$. Then this means that there is $s$ such that $0 \neq y_{s} \in g r_{\text {geo }}^{s^{\prime}}(X)$ with $s-s^{\prime}=0 \bmod (2 p-2)$. Of course if $s \neq k$, then $k-s^{\prime} \neq 0 \bmod (2 p-2)$.

To study the difference of $F_{g e o}^{*}(X)$ and $F_{\text {top }}^{*}(X(\mathbb{C}))$, we consider AHss $E_{r}^{*, *^{\prime}}(B P)$ converging to $B P^{*}(X)$. Suppose that

$$
\left[v_{1} \otimes x\right] \in B P^{*^{\prime}} \otimes H^{*}(X(\mathbb{C})) \cong E(B P)_{2}^{*, 0}
$$

is an permanent cycle, but $[x] \in H^{*}(X(\mathbb{C}))$ itself is not (i.e., $d_{r}(x) \neq 0$ for some $r)$. Let $x^{\prime} \in B P^{*}(X(\mathbb{C}))$ be a corresponding element for $\left[v_{1} \otimes x\right]$ in $E_{\infty}^{*, *^{\prime}}$

Lemma 3.5. Let $x \in H^{2 *}(X(\mathbb{C}))$ and $x^{\prime} \in B P^{*^{\prime}}(X(\mathbb{C}))$ be elements with the assumption above. Suppose that

$$
0 \neq x^{\prime} \in B P^{*^{\prime}}(X(\mathbb{C})) \otimes_{B P^{*}} \mathbb{Z}\left[v_{1}, v_{1}^{-1}\right] \cong \tilde{K}(1)^{*}(X(\mathbb{C}))
$$

and that $x^{\prime} \in B P^{*^{\prime}}(X(\mathbb{C})) \otimes_{B P^{*}} \mathbb{Z}$ is in the image of the Totaro cycle map

$$
C H^{*^{\prime}}(X) \rightarrow B P^{2 *^{\prime}}(X(\mathbb{C})) \otimes_{B P^{*}} \mathbb{Z} .
$$

Then $0 \neq x^{\prime} \in g r_{\text {top }}^{2 *}(X(\mathbb{C}))$, but $\left.0 \neq x^{\prime} \in g r_{\text {geo }}^{2(*-p+1)}(X)\right)$.

Proof. This case $*^{\prime}=*-(p-1)$ in the above arguments. Let $x \in H^{2 i}(X(\mathbb{C}))$. In fact $x^{\prime} \in \operatorname{Im}\left(C H^{i-p+1}(X)\right)$ and $0 \neq x^{\prime} \in g r_{\text {geo }}^{2(i-p+1)}(X(\mathbb{C}))$, but $0 \neq x^{\prime}=\left[v_{1} \otimes x\right] \in$ $g r_{\text {top }}^{2 i}(X(\mathbb{C}))$.

Next we consider the cases $g r_{\gamma}^{*}(X) \cong g r_{t o p}(X(\mathbb{C}))$. From the Atiyah theorem (Lemma 2.4), the following lemma is immediate.

Lemma 3.6. Suppose $(*)$ and suppose that the infinity term $E_{\infty}^{2 *, 0}(\tilde{K}(1))$ (of the AHss for $\tilde{K}(1)^{*}(X(\mathbb{C}))$ ) is generated by Chern classes in $H^{*}(X)$ for all $* \geq N$. Then for all $* \geq N$, we have

$$
g r_{\gamma}^{2 *}(X) \cong E_{\infty}^{2 *, 0}\left(\tilde{K}(1)^{*}(X(\mathbb{C}))\right) \quad \text { for all } * \geq N .
$$

Lemma 3.7. (Lemma 2.8 in [Ya3]) Suppose $(*)$ and that $H^{*}(X(\mathbb{C}))$ is generated by Chern classes. Then we have

$$
C H^{*}(X) \cong H^{*}(X(\mathbb{C})) \quad \text { for } * \leq p-1 .
$$

Moreover if $X(\mathbb{C})$ is simply connected (resp. 3-connected), then we have the isomorphisms for $* \leq p($ resp. $* \leq p+1)$

$$
C H^{*}(X) \otimes \mathbb{Z}_{p} \cong H^{2 *}\left(X(\mathbb{C}) ; \mathbb{Z}_{p}\right) .
$$

Proof. By the assumption, we see

$$
g r_{\gamma}^{2 *}(X) \cong g r_{\text {geo }}^{2 *}(X) \cong g r_{\text {top }}^{2 *}(X(\mathbb{C})) .
$$

To compute the last graded ring, we consider AHss

$$
E_{2}^{*, *^{\prime}}(\tilde{K}(1)) \cong H^{*}\left(X ; \tilde{K}(1)^{*^{\prime}}\right) \Longrightarrow \tilde{K}(1)^{*}(X(\mathbb{C})) .
$$


Here $\tilde{K}(1)^{*} \cong \mathbb{Z}\left[v_{1}, v_{1}\right]$ with $\left|v_{1}\right|=-2 p+2$. It is well known that the first non zero differential is

$$
d_{2 p-1}(x)=v_{1} \otimes Q_{1}(x) \quad \bmod (p) .
$$

So each element in $H^{2 *}(X(\mathbb{C}))$ is not tergent of any differential $d_{r}$ when $* \leq p-1$. (Of course $d_{r}(x)=0$ for Chern classes $x$.) Hence we have

$$
g r_{\text {top }}^{2 *}(X(\mathbb{C})) \cong H^{2 *}(X(\mathbb{C})) \quad \text { for } * \leq p-1 .
$$

Similarly, considering AHss converging to $A \tilde{K}(1)^{*, *^{\prime}}(X)$, we have the isomorphism

$$
g r_{g e o}^{2 *}(X) \cong C H^{2 *}(X) \quad \text { for } * \leq p-1 .
$$

Here we use the fact $E_{2}^{2 *, *, 0}(A \tilde{K}(1)) \cong C H^{*}(X)$. Thus the isomorphism of the geometric and toplogical filtrations, gives the first statements.

From the isomorphism

$$
H^{1,1}(X ; \mathbb{Z} / p) \cong H^{1}(X(\mathbb{C}) ; \mathbb{Z} / p)=0 .
$$

we see that $H^{1,1}(X ; \mathbb{Z})$ is $p$-divisible. Since the image of the differential of $p$-divisible elements are also $p$-divisible,

$$
\begin{gathered}
H^{2 p}(X(\mathbb{C})) \cong g r_{\text {top }}^{2 p}(X) \\
\cong g r_{\text {geo }}^{2 p}(X) \cong C H^{2 p}(X) /(p-\text { divisible }) .
\end{gathered}
$$

Hence we have the second isomorphism. The third isomorphism is seen similarly.

Remark. The first statement in the above lemma is also proved by the RiemannRoch formula without denominators, namely, the composition map

$$
C H^{i}(X) \rightarrow g r_{g e o}^{i}(X) \stackrel{c_{i}}{\rightarrow} C H^{i}(X)
$$

is multiplication by $(-1)^{i-1}(i-1)$ !. Hence we get $C H^{i}(X) \cong g r_{g e o}^{i}(X)$ for $i \leq p$. Moreover we know that $C H^{i}(X)$ is represented by the $i$-th Chern class $c_{i}(\xi)$ for some bundle $\xi$.

Remark. The assumption of Lemma 2.8 in [Ya3] is not sufficient, and it should be changed as above.

\section{CLASSIFYing SPACES $B G$ FOR FINITE GROUPS}

Let $G$ be a compact Lie group (e.g., a finite group) and $G_{k}$ be the corresponding algebraic group over an algebraically closed field $k$ in $\mathbb{C}$. Then by Merkurjev and Totaro ([To]), we have the isomorphisms

$$
\text { (1.1) } K_{\text {alg }}^{0}\left(B G_{k}\right) \cong R\left(G_{k}\right)^{\wedge} \cong R(G)^{\wedge} \cong K_{\text {top }}^{0}(B G),
$$

where $R\left(G_{k}\right)^{\wedge}$ (resp. $R(G)^{\wedge}$ ) is the $k$-representation (resp. complex representation) ring completed by the augmentation ideal and $K_{\text {alg }}^{0}\left(B G_{k}\right)\left(\operatorname{resp} . K_{\text {top }}^{0}(B G)\right)$ is the $K$-theory generated by $k$-bundles (resp. complex bundles) of the classifying space $B G_{k}$ (resp. $\left.B G\right)$.

When $k$ is algebraically closed, we write $B G_{k}$ by $B G$ simply. For Section 4-6, we assume $k$ is algebraically closed.

In this section, we consider cases that $G$ are finite groups. At first, we consider the case $G=\mathbb{Z} / p^{r}$. Then $H^{*}(B G) \cong \mathbb{Z}[y] /\left(p^{r} y\right),|y|=2$ and $y_{1}=c_{1}(e)$ for a nonzero linear representation $e$. So all three filtrations are the same. The similar fact holds for its product. 
Theorem 4.1. ( $p=2, r=1$ case by Atiyah [At]) Let $q=p^{r}$ and $G=\oplus^{n} \mathbb{Z} / q$. Then

$$
g r_{\text {top }}^{*}(B G) \cong \mathbb{Z}\left[y_{1}, \ldots, y_{n}\right] /\left(q y_{i}, y_{i}^{q} y_{j}-y_{i} y_{j}^{q}\right) .
$$

Hence the three filtrations are the same.

Proof. Let $Q_{0}^{\prime}=\beta_{q}$ be the higher Bockstein. The integral cohomology is isomorphic to a subring of the $\bmod q$ cohomology

$$
H^{*}(B G) \subset H^{*}(B G ; \mathbb{Z} / q), \quad \text { when } *>0 .
$$

Here $H^{*}(B G ; \mathbb{Z} / q) \cong \mathbb{Z} / q\left[y_{1}, \ldots, y_{n}\right] \otimes \Lambda\left(x_{1}, \ldots, x_{n}\right)$ with $Q_{0}^{\prime}\left(x_{i}\right)=y_{i}$, and we know

$$
H^{*}(B G) \cong \mathbb{Z} / q\left[y_{1}, \ldots, y_{n}\right]\left\{Q_{0}^{\prime}\left(x_{i_{1}} \ldots x_{i_{s}}\right) \mid 1 \leq i_{1}<\ldots, i_{s} \leq n\right\}
$$

with $Q_{0}^{\prime}\left(x_{i_{1}} \ldots x_{i_{s}}\right)=\sum_{k}(-1)^{k-1} y_{i_{k}} x_{i_{1}} \ldots \hat{x}_{i_{k}} \ldots x_{i_{s}}$.

We consider the AHss converging to $\tilde{K}(1)^{*}(B G)$. We define the weight degree for elements in this AHss by

$$
w\left(v_{1}\right)=0, \quad w\left(y_{i}\right)=0, \quad w\left(x_{i}\right)=1
$$

so that $w\left(Q_{0}^{\prime}\left(x_{i_{1}} \ldots x_{i_{s}}\right)\right)=s-1$. We will prove

$$
\text { (1) }(\text { weight }=0) \cap E_{2 q}^{*, *^{\prime}} \cong \mathbb{Z} / q\left[y_{1}, \ldots, y_{n}\right] /\left(y_{i}^{q} y_{j}-y_{i} y_{j}^{q}\right) \quad \text { for } *>0 \text {, }
$$

(2) $\quad($ weight $=1) \cap E_{2 q}^{*, *^{\prime}}=0$.

Then we can prove this theorem by the following arguments.

We consider the AHss converging to the motivic $A \tilde{K}(1)^{*}(B G)$. The weight $w(x)$ of an element $x \in H^{*, *^{\prime}}(X: \mathbb{Z} / q)$ is defined as $2 *^{\prime}-*$. Since $x_{i} \in H^{1,1}(B G ; \mathbb{Z} / q)$ and $y_{i} \in H^{2,1}(B G ; \mathbb{Z} / q)$, their weights are in fact $w\left(x_{i}\right)=1$ and $w\left(y_{i}\right)=0$. The degree of the motivic AHss is

$$
\operatorname{deg}\left(d_{2 r-1}\right)=(2 r-1, r-1,-2(r-1)) \quad \text { with }(r-1)=0 \bmod (p-1),
$$

namely, $w\left(d_{2 r-1}\right)=-1$ which means

$$
d_{2 r-1}(\text { weight }=s)=(\text { weight }=s-1) .
$$

From $(2)$, (weight $=0$ )-parts are not a target of any diffrential $d_{2 r-1}$ for $r>q$. By the naturality of realization map from the motivic AHss to the usual AHss, we get the same fact for the AHss for $\tilde{K}(1)^{*}(B G)$. Since $\tilde{K}(1)^{*}(B G)$ is generated by only weght $=0$ elements, we have the theorem.

The first nonzero differential is known $d_{2 q-1}\left(x_{i}\right)=v_{1}^{1+p+\ldots+p^{r-1}} y_{i}^{q}[$ Ya2]. We see (1) from

$$
d_{2 q-1}\left(Q_{0}^{\prime}\left(x_{1} x_{2}\right)\right)=d_{2 q-1}\left(y_{1} x_{2}-y_{2} x_{1}\right)=y_{1} y_{2}^{q}-y_{1}^{q} y_{2}
$$

Now we prove $(2)$. Let $x \in \operatorname{Ker}\left(d_{2 s-1}\right)$ and $x=\sum a_{i j} Q_{0}^{\prime}\left(x_{i} x_{j}\right)$. Then $\left(\right.$ since $d_{r}$ is a derivation)

$$
d_{2 q-1}(x)=\sum a_{i j}\left(y_{i} y_{j}^{q}-y_{i}^{q} y_{j}\right)=0 \quad \text { in } \mathbb{Z} / q\left[y_{1}, \ldots, y_{n}\right] .
$$

Here we consider them in $\bmod \left(x_{i}, y_{i} \mid i \geq 4\right)$. Then we see $a_{12}=a_{12}^{\prime} y_{3}$ and we see (by dividing $y_{1} y_{2} y_{3}$ )

$$
a_{12}^{\prime}\left(y_{1}^{q-1}-y_{2}^{q-1}\right)+a_{23}^{\prime}\left(y_{2}^{q-1}-y_{2}^{q-1}\right)+a_{31}^{\prime}\left(y_{3}^{q-1}-y_{1}^{q-1}\right)=0 .
$$

This implies that $a_{12}^{\prime} \in \operatorname{ideal}\left(y_{1}^{q-1}, y_{2}^{q-1}, y_{3}^{q-1}\right)$. Moreover we see that $a_{12}$ contains $y_{3}^{q}$. Similarly $a_{23}, a_{13}$ contains $y_{1}^{q}$ and $y_{2}^{q}$ respectively. 
On the other hand, we see

$$
\begin{gathered}
d_{2 q-1}\left(Q_{0}^{\prime}\left(x_{1} x_{2} x_{3}\right)\right)=d_{2 q-1}\left(\sum y_{1} x_{2} x_{3}\right) \\
=\sum y_{1} y_{2}^{q} x_{3}-\sum y_{1} x_{2} y_{3}^{q}=\sum y_{1} y_{2}^{q} x_{3}-\sum y_{3} x_{1} y_{2}^{q} \\
=\sum y_{2}^{q}\left(y_{1} x_{3}-y_{3} x_{1}\right)=-\sum y_{1}^{q} Q_{0}^{\prime}\left(x_{2} x_{3}\right)
\end{gathered}
$$

Taking off $a^{\prime \prime} d_{2 r-1} Q_{0}^{\prime}\left(x_{1} x_{2} x_{3}\right)$ for some adequate $a^{\prime \prime} \in \mathbb{Z} / q\left[y_{1}, \ldots, y_{n}\right]$, we can prove (2).

Recall that a group $G$ is called an extraspecial $p$-group if its center $Z(G) \cong \mathbb{Z} / p$ and there is a central extension

$$
0 \rightarrow \mathbb{Z} / p \rightarrow G \rightarrow \oplus^{2 n} \mathbb{Z} / p \rightarrow 0
$$

For each prime $p$, such groups have only two types, namely, $p_{+}^{1+2 n}, p_{-}^{1+2 n}$. (e.g., $2_{+}^{1+2} \cong D_{8}$ the dihedral group (of order 8 ), $p_{-}^{1+2} \cong Q_{8}$ the quaternion group). We here only write down the case $p_{+}^{1+2}$ for $p \geq 3$. The cohomology is known ([Ya1,3])

$$
H^{\text {even }}(B G) \cong(Y \oplus B) \otimes \mathbb{Z}\left[c_{p}\right] /\left(p^{2} c_{p}\right)
$$

where $Y=\mathbb{Z}\left[y_{1}, y_{2}\right] /\left(p y_{i}, y_{1} y_{2}^{p}-y_{1}^{p} y_{2}\right), B=\mathbb{Z} / p\left\{c_{2}, \ldots, c_{p-1}\right\}$ and $\left|y_{i}\right|=c_{1}\left(e_{i}\right)$ and $c_{i}=c_{i}(\xi)$ for some linear representations $e_{i}$ and $p$-dimensional representation $\xi$. Hence the even dimensional part of this cohomology is generated by Chern classes and all three filtrations are the same. The odd degree part is

$$
H^{\text {odd }}(B G) \cong Y \otimes \mathbb{Z} / p\left[c_{p}\right]\left\{a_{1}, a_{2}\right\} /\left(y_{2} a_{1}-y_{1} a_{2}, y_{2}^{p} a_{1}-y_{1}^{p} a_{2}\right) \quad\left|a_{i}\right|=3 .
$$

Theorem 4.2. Let $G=p_{+}^{1+2}$ and $p \geq 3$. Then

$$
g r_{\text {top }}^{*}(B G) \cong Y \oplus\left(\mathbb{Z}\left\{c_{p}\right\} \oplus B\right) \otimes \mathbb{Z}\left[c_{p}\right] /\left(p^{2} c_{p}\right) .
$$

Proof. We know the Milnor cohomology operation

$$
v_{1}^{-1} d_{2 p-1}=Q_{1}: H^{\text {odd }}(B G) \rightarrow H^{\text {even }}(B G)
$$

is injective and $Q_{1}\left(a_{i}\right)=y_{i} c_{p}$. Hence we see

$$
\begin{aligned}
\operatorname{gr} \tilde{K}(1)^{*}(B G) & \cong E_{\infty}^{*, *^{\prime}} \cong \tilde{K}(1)^{*} \otimes H^{\text {even }}(B G) /\left(Q_{1} H^{\text {odd }}(B G)\right) \\
& \cong \tilde{K}(1)^{*} \otimes H^{\text {even }}(B G) /\left(y_{i} c_{p}\right) .
\end{aligned}
$$

When $p \geq 5$, the groups of $\operatorname{rank}_{p} G=2$ are classified by Blackburn. When groups are of class 2 (i.e., $[G,[G, G]]=1$ ), cohomology rings are generated by Chern classes ([Le-Ya],[Ya1]), and hence all three filtrations are the same. Define the class $3 p$-group (i.e., $[G,[G, G]] \neq 1$ ) by

$$
\begin{gathered}
G(4,1)=\langle a, b, c| a^{p}=b^{p}=c^{p+1}=[b, c]=1, \\
\left.\left[a, b^{-1}\right]=c^{p},[a, c]=b\right\rangle .
\end{gathered}
$$

Let $G=G(4,1)$. Then there is an element $x_{p+1} \in H^{2 p+2}(B G)$ [Le-Ya],[Ya] such that it is permanent in AHss for $\tilde{K}(1)^{*}(B G)$ and $x_{p+1}$ is not represented by Chern class. But all elemnts in $H^{\text {even }}(B G)$ is represented by transfers of Chern classes [Ya]. Of course Chow rings have the transfer map. Hence we have

Theorem 4.3. Let $p \geq 5$ and $G=G(4,1)$. Then $g r_{\text {top }}^{*}(B G) \cong g r_{\text {geo }}^{*}(B G)$ but $g r_{\gamma}^{i}(B G) ¥ g r_{\text {geo }}^{i}(B G)$ for $i=4,2 p+2$. 
Proof. The first isomorphism follows from that all elements in $H^{\text {even }}(B G)$ is represented by transfer of Chern classes. The second statement follows from $x_{p+1}$ is not represented by Chern classes and the element $x_{p+1} \in E_{\infty}^{2 p+2,0}$ represents a nonzero element in $g r_{\gamma}^{4}(B G)$ from Lemma 3.4.

\section{CONNECTED GROUPS WITH $p=2$}

Throughout this section, let $p=2$. At first we consider the case $G=O_{n}$. The mod 2 cohomology of the classifying space $B O_{n}$ of the $n$-th orthogonal group is

$$
H^{*}\left(B O_{n} ; \mathbb{Z} / 2\right) \cong H^{*}\left((B \mathbb{Z} / 2)^{n} ; \mathbb{Z} / 2\right)^{S_{n}} \cong \mathbb{Z} / 2\left[w_{1}, \ldots, w_{n}\right]
$$

where $S_{n}$ is the $n$-th symmetry group, $w_{i}$ is the Stiefel-Whiteney class which restricts the elementary symmetric polynomial in $\mathbb{Z} / 2\left[x_{1}, \ldots, x_{n}\right]$. Each element $w_{i}^{2}$ is represented by Chern class $c_{i}$ of the induced representation $O_{n} \subset U_{n}$. Let us write $w_{i}^{2}$ by $c_{i}$.

Recall the Milnor operation $Q_{i}$ which is defined $Q_{0}=\beta$ and $Q_{i}=\left[Q_{i-1}, P^{p^{i-1}}\right]$. Let us write by $Q(i)$ the exteria algebra $\Lambda\left(Q_{0}, \ldots, Q_{i}\right)$. W.S.Wilson ([Wi],[Ko-Ya]) found a good $Q(i)$-module decomposition for $B O_{n}$, namely,

$$
H^{*}\left(B O_{n} ; \mathbb{Z} / 2\right)=\oplus_{i=-1} Q(i) G_{i} \quad \text { with } Q_{0} \ldots Q_{i} G_{i} \in \mathbb{Z} / 2\left[c_{1}, \ldots, c_{n}\right] .
$$

Let us write by $P(n)^{*}=B P^{*} /\left(p, \ldots, v_{i-1}\right)$. The $B P^{*}$-theory is then computed

$$
\operatorname{grBP} P^{*}(B G) / p \cong \oplus P(i+1)^{*} Q_{0} \ldots Q_{i} G_{i} .
$$

Hence we have $K(1)^{*}(B G) \cong P(1)^{*}\left(G_{-1} \oplus Q_{0} G_{0}\right)$.

Moreover, by Wilson, it is known that

$$
B P^{*}\left(B O_{n}\right) \cong B P^{*}\left[\left[c_{1}, \ldots, c_{n}\right]\right] /\left(c_{1}-c_{1}^{*}, \ldots, c_{n}-c_{n}^{*}\right)
$$

where $c_{i}^{*}$ is the conjugation of $c_{i}$. Hence $\tilde{K}(1)^{*}(B G)$ is generated by Chern classes from $H^{*}(B G)$. Thus from Lemma 2.4, all filtrations are same.

Here $G_{k-1}$ is quite complicated (see for details [Wi]), namely, it is generated by symmetric functions

$$
\sum x_{1}^{2 i_{1}+1} \ldots x_{k}^{2 i_{k}+1} x_{k+1}^{2 j_{1}} \ldots x_{k+q}^{2 j_{q}}, \quad k+q \leq n,
$$

with $0 \leq i_{1} \leq \ldots \leq i_{k}$ and $0 \leq j_{1} \leq \ldots \leq j_{q}$; and if the number of $j$ equal to $j_{u}$ is odd, then there is some $s \leq k$ such that $2 i_{s}+2^{s}<2 j_{u}<2 i_{s}+2^{s+1}$.

Thus when $k \leq 1$, there is not above $j_{u}$, that means numbers of $j=j_{u}$ are always even.

Theorem 5.1. Let $G=O_{n}$. Then all three fitrations are the same, and $\operatorname{gr}_{\text {top }}^{*}(B G) \cong$ $A \oplus B / 2$ with $\left(y_{i}=x_{i}^{2}\right.$ so that $\left.\sum y_{1}=c_{1}\right)$

$$
A=\mathbb{Z}\left\{\sum\left(y_{1} y_{2}\right)^{j_{1}} \ldots\left(y_{2 s-1} y_{2 s}\right)^{j_{s}}\right\} \quad B=\mathbb{Z}\left\{\sum y_{1}^{i}\left(y_{2} y_{3}\right)^{j_{1}} \ldots\left(y_{2 s} y_{2 s+1}\right)^{j_{s}}\right\} .
$$

(Note $A / 2=G_{-1}$ and $B / 2=Q_{0} G_{0}$.)

Example. When $G=O_{2}$, we have the isomorphism $g r_{\text {top }}^{*}(B G) \cong \mathbb{Z}\left[c_{2}\right] \oplus$ $\mathbb{Z} / 2\left[c_{1}\right]$.

When $G=S O_{\text {odd }}$, (since $S O_{\text {odd }} \times \mathbb{Z} / 2 \cong O_{\text {odd }}$ ), the situations are same. Let $G=S O_{2 n}$. Then from Field, we have ([Fi], [Ma-Vi], [In-Ya])

$$
\begin{gathered}
C H^{*}(B G) \cong \mathbb{Z}\left[c_{2}, \ldots c_{2 n}\right]\left\{y_{2 n}\right\} \oplus C H^{*}\left(B O_{2 n}\right) /\left(c_{1}\right), \\
B P^{*}(B G) \cong B P^{*}\left[c_{2}, \ldots, c_{2 n}\right]\left\{y_{2 n}\right\} \oplus B P^{*}\left(B O_{2 n}\right) /\left(F_{1}\right)
\end{gathered}
$$


where $F_{1}=\operatorname{ker}\left(B d e t^{*}\right)$ and $y_{2 n}^{2}=(-1)^{n} 2^{2 n-2} c_{2 n}$. Hence

$$
y_{2 n}=(-1)^{*} 2^{n-1} w_{2 n} \in H^{*}(B G)_{(2)} .
$$

By Field, it is shown that just $(n-1) ! y_{2 n}$ (for $\left.n>2\right)$ is represented by Chern classes (Theorem 8, Corollary 2 in [Fi]). Thus we have

Theorem 5.2. Let $G=S O_{2 n}$ and $n \geq 3$. Then

$$
g r_{\text {top }}^{*}(B G)=g r_{\text {geo }}^{*}(B G) \cong \mathbb{Z}\left[c_{2}, c_{4}, \ldots, c_{2 n}\right]\left\{y_{2 n}\right\} \oplus g r_{\text {top }}^{*}\left(B O_{2 n}\right) /\left(c_{1}\right) .
$$

However we have $g r_{\gamma}^{2 n}(B G) \neq g r_{\text {geo }}^{2 n}(B G)$.

We note when $G=S_{4}$, all the three filtrations are same, since $y_{4}$ is represented by Chern classes.

Proposition 5.3. Let $G=S O_{2(p+1)}$ and $p \neq 2$. Then

$$
g r_{\gamma}^{*}(B G) \cong \mathbb{Z}_{(p)}\left[c_{2}, \ldots, c_{2 p+2}\right] \otimes\left(\mathbb{Z}_{(p)}\left\{1, y^{\prime}\right\} \oplus \mathbb{Z} / p\{y\}\right)
$$

with $\left|y^{\prime}\right|=2(p+1)$ and $|y|=4$.

We consider the exceptional Lie group $G_{2}$. Let $G=G_{2}$. Its $\bmod (2)$ cohomology is well known

and integral cohomology is

$$
H^{*}(B G ; \mathbb{Z} / 2) \cong \mathbb{Z} / 2\left[w_{4}, w_{6}, w_{7}\right]
$$

$$
H^{*}(B G) \cong \mathbb{Z}\left[w_{4}, c_{6}\right] \otimes\left(\mathbb{Z}\{1\} \oplus \mathbb{Z} / 2\left[w_{7}\right]\left\{w_{7}\right\}\right) .
$$

We can compute the AHss for $B P^{*}(B G)$ ([Ko-Ya], [Sc-Ya])

$$
\operatorname{gr} B P^{*}(B G) \cong \mathbb{Z}\left[c_{4}, c_{6}\right] \otimes\left(B P^{*}\left\{1,2 w_{4}\right\} \oplus P(3)^{*}\left[c_{7}\right]\left\{c_{7}\right\}\right) .
$$

Here we can show the element $\left\{2 w_{4}\right\}$ is represented by a Chern class $c_{2}^{\prime}$. We see $\left.\tilde{K}(1)^{*}(B G) \cong \tilde{K}(1)^{*}\left[c_{4}, c_{6}\right] \otimes\left\{1,2 w_{4}\right\}\right)$, and $([\mathrm{Ya} 2],[\mathrm{Gu}])$

$$
C H^{*}(B G) \cong B P^{*}(B G) \otimes_{B P^{*}} \mathbb{Z} \cong \mathbb{Z}\left[c_{2}^{\prime}, c_{4}, c_{6}, c_{7}\right] /\left(\left(c_{2}^{\prime}\right)^{2}-4 c_{4}, 2 c_{7}\right) .
$$

Theorem 5.4. Let $G=G_{2}$. Then all three filtrations are the same

$$
g r_{\text {top }}^{*}(B G) \cong C H^{*}(B G) /\left(c_{7}\right) \cong \mathbb{Z}\left[c_{2}^{\prime}, c_{4}, c_{6}\right] /\left(\left(c_{2}^{\prime}\right)^{2}-4 c_{4}\right) .
$$

Next we study the case $G=\operatorname{Spin}_{7}$. Its $\bmod (2)$ cohomology is

$$
H^{*}(B G ; \mathbb{Z} / 2) \cong \mathbb{Z} / 2\left[w_{4}, w_{6}, w_{7}, w_{8}\right] .
$$

The infinity term of the AHss for $B P^{*}(B G)$ is still computed

$$
\begin{gathered}
\operatorname{grBP} P^{*}(B G) \cong \mathbb{Z}\left[c_{4}, c_{6}\right] \otimes\left(B P^{*}\left[c_{8}\right]\left\{1,2 w_{4}, 2 w_{8}, 2 w_{4} w_{8}, v_{1} w_{8}\right\}\right. \\
\left.\oplus P(3)^{*}\left[c_{7}\right]\left\{c_{7}\right\} \oplus P(4)^{*}\left[c_{7}, c_{8}\right]\left\{c_{7} c_{8}\right\}\right) .
\end{gathered}
$$

Hence we see

$$
\operatorname{gr} \tilde{K}(1)^{*}(B G) \cong \tilde{K}(1)^{*}\left[c_{4}, c_{6}, c_{8}\right]\left\{1,2 w_{4}, 2 w_{8}, 2 w_{4} w_{8}, v_{1} w_{8}\right\} .
$$

Here it is known that $2 w_{4}, 2 w_{8}, 2 w_{4} w_{8}$ are represented by Chern classes, and write them $c_{2}^{\prime}, c_{4}^{\prime}, c_{6}^{\prime}$. But it is proved (Theorem 6.2 in [Sc-Ya]) that $v_{1} w_{8}$ is not represented by (transfer) of Chern classes while it is in the image of cycle map. Let $c l(\xi)=\left[v_{1} w_{8}\right]([\mathrm{Gu}]$, Lemma 9.6 in $[\mathrm{Ya}], \S 9$ in $[\mathrm{Ka}-\mathrm{Te}-\mathrm{Ya}])$. Totraro's conjecture also holds this case

$$
\begin{gathered}
C H^{*}(B G) \cong B P^{*}(B G) \otimes_{B P^{*}} \mathbb{Z} \\
\cong \mathbb{Z}\left[c_{4}, c_{6}, c_{8}\right] \otimes\left(\mathbb{Z}\left\{1, c_{2}^{\prime}, c_{4}^{\prime}, c_{6}^{\prime}\right\} \oplus \mathbb{Z} / 2\{\xi\} \oplus \mathbb{Z} / 2\left[c_{7}\right]\left\{c_{7}\right\}\right)
\end{gathered}
$$

with $|\xi|=6$. Moreover, we can prove 
Lemma 5.5. Let $G=\operatorname{Spin}_{7}$. Any element $x \in B P^{*}(B G)$ such that

$$
0 \neq x=\left[v_{1} w_{8}\right] a \in B P^{*}(B P) \quad \text { with } a \in \mathbb{Z}\left[c_{4}, c_{6}, c_{8}\right],
$$

can not be generated by Chern classes of $B P^{*}$-theory.

Proof. Let $N=Z(G) \cong \mathbb{Z} / 2$ be the center of $G$ and $N \oplus A$ is a maximal elementary abelian 2-subgroup of $G$, so $A \cong(\mathbb{Z} / 2)^{3}$. A representation $\xi$ of $G$ is said to be a spin representation, if $\xi \mid N \neq 0$. For a nonspin representation $\eta$, we know the total Chern class

$$
\left.\eta\right|_{N \oplus A}=\left.\eta\right|_{A} \in B P^{*}\left[c_{4}, c_{6}, c_{7}\right]
$$

For a spin representation $\chi$, we have

$$
\left.(\chi)\right|_{N}=(1+u)^{s} \in B P^{*}(B N) \cong B P^{*}[u] /([2](u)) \quad|u|=2
$$

where $[2](u)=2 u+v_{1} u^{2}+\ldots$ is the 2 -th product of the $B P^{*}$-formal group laws. Here we note $s=8 s^{\prime}$ since $\left.c_{8}\right|_{N}=u^{8}$. It is known that $\left.v_{1} w_{8}\right|_{N}=v_{1} u^{4}$ [Sc-Ya]. Then

$$
\left.c(\chi)\right|_{N}=\left(1+8 u+28 u^{2}+\ldots+u^{8}\right)^{s} .
$$

Here we can compute

$$
8 u=4 v_{1} u^{2}=2 v_{1}^{2} u^{3}=v_{1}^{3} u^{4}, \quad 28 u^{2}=14 v_{1} u^{3}=7 v_{1}^{2} u^{4}, \ldots
$$

Thus we see that $v_{1} u^{4}$ is not represented by the restriction of Chern classes. (However $v_{1}^{2} u^{4}$ has its possibily, infact $\left|v_{1} w_{8}\right|=4$ and it is represented by the Chern class $c_{2}$.)

Of course $c(\chi \oplus \eta)=c(\chi) c(\eta)$, we get the lemma.

Theorem 5.6. Let $G=\operatorname{Spin}_{7}$. Then

$$
\begin{gathered}
g r_{\text {top }}^{*}(B G) \cong \mathbb{Z}\left[c_{4}, c_{6}, w_{8}\right]\left\{1, c_{2}^{\prime}\right\}, \\
g r_{\alpha}^{*}(B G) \cong \mathbb{Z}\left[c_{4}, c_{6}, c_{8}\right]\left(\mathbb{Z}\left\{1, c_{2}^{\prime}, c_{4}^{\prime}, c_{6}^{\prime}\right\} \oplus \mathbb{Z} / 2\{\xi\}\right)
\end{gathered}
$$

where $\operatorname{deg}(\xi)=6($ resp. $=4)$ if $\alpha=$ geo $($ if $\alpha=\gamma)$.

Recall that $2_{+}^{1+2 n}$ is the extraspecial 2-group, which is isomorphic to the central product of $n$-copies of the dihedral group $D_{8}$ of order 8 . Let $G=2_{+}^{1+6}$. There is an inclusion $i: G \subset \operatorname{Spin}_{7}$ and its induced map $i^{*}: H^{*}\left(B \operatorname{Spin}_{7} ; \mathbb{Z} / 2\right) \rightarrow H^{*}(B G ; \mathbb{Z} / 2)$ is also injective by Quillen $[\mathrm{Qu}]$. Let $j: \mathbb{Z} / 2 \cong Z(G) \subset G$. Then it is know [Qu], $[$ Sc-Ya $] j^{*} i^{*}\left(w_{8}\right)=u^{4} \in \mathbb{Z}[u] /(2 u) \subset H^{*}(B Z(G))$. Hence we have in $\tilde{K}(1)^{*}$-theory

$$
j^{*} i^{*}\left(v_{1} w_{8}\right)=v_{1} u^{4} \neq 0 \in \tilde{K}(1)^{*}(B Z(G)) \cong \tilde{K}(1)^{*}[u] /\left(2 u-v_{1} u^{2}\right) .
$$

This element $v_{1} \otimes w_{8}$ is not generated by Chern classes also in $H^{*}(B G)$. Hence we have

Corollary 5.7. Let $G=2_{+}^{1+6}$. Then there is an element $x \in A \tilde{K}(1)^{*}(B G)$ such that

$$
0 \neq x \in g r_{\gamma}^{4}(B G), \quad x=\xi \in g r_{g e o}^{6}(B G), \quad \text { and } \quad x=w_{8} \in g r_{\text {top }}^{8}(B G) .
$$




\section{CONNECTED GROUPS FOR $p$ ODD}

In this section, we assume $p \geq 3$. At first we consider the case $G=P G L_{p}$. Its $\bmod p$ cohomolgy is given by Vistoli and Kameko-Yagita ([Vi], [Ka-Ya]), namely, there is a short exact sequence

$$
0 \rightarrow M / p \rightarrow H^{*}(B G) \rightarrow N \rightarrow 0
$$

where $M \cong Z\left[x_{4}, x_{6}, \ldots, x_{2 p}\right]$ additively (but not as rings), and $N \cong N^{\prime} \otimes \Lambda\left(Q_{0}, Q_{1}\right)\left\{u_{2}\right\}$, $\left|u_{2}\right|=2$ for some $\mathbb{Z} / p$-module $N^{\prime}$. The $B P$-theory $B P^{*}(B G)$ is also studied There is a short exact sequence

$$
0 \rightarrow B P^{*} \otimes M \rightarrow \operatorname{grBP} P^{*}(B G) \rightarrow N^{\prime \prime} \rightarrow 0
$$

where $g r N^{\prime \prime} \cong P(3)^{*} \otimes N^{\prime} \otimes Q_{0} Q_{1}(u)$. Therefore we see $g r^{*} \tilde{K}(1)^{*}(B G) \cong M$ additively. Totaro's conjecture also holds this case. Thus we have

Theorem 6.1. Let $G=P G L_{p}$. Then

$$
g r_{\text {top }}^{*}(B G) \cong g r_{\text {geo }}^{*}(B G)(\cong M \text { additively }) .
$$

When $p=3$, it is known (page 2274 in $[\mathrm{Ka}-\mathrm{Ya}]$ ) that $M / 3 \cong \mathbb{Z} / 3\left[c_{2}, c_{3}, c_{6}\right] /\left(c_{2}^{3}-\right.$ $\left.c_{6}^{2}\right)$. Hence the gamma filtration is the same when $p=3$. However, for $p \geq 5$, it seems unknown that $M$ above is generated by Chern classes or not.

For exceptional Lie groups, we can compute $B P^{*}(B G)$ except for $(G, p)=$ $\left(E_{8}, p=3\right)$. So we know $g r_{t o p}^{*}(B G)$, but it seems not so easy to compute $C H^{*}(B G)$ now, and $g r_{g e o}^{*}(B G)$ seems unknown. For example, when $G=F_{4}$ we can compute $B P^{*}(B G)$. The $\bmod (3)$ cohomology is generated by $x_{4}, x_{8}, x_{9}, x_{20}, x_{21}, \ldots$ (by Toda). The $B P$-theory is also computed

$$
\operatorname{gr} B P^{*}(B G) \cong B P^{*}\left[c_{18}, c_{24}\right]\left\{1,3 x_{4}\right\} \oplus B P^{*} \otimes E \oplus P(3)^{*}\left[x_{26}\right]\left\{x_{26}\right\}
$$

where $E=\mathbb{Z}\left[x_{4}, x_{8}\right]\left\{a b \mid a, b \in\left\{x_{4}, x_{8}, x_{20}\right\}\right\}$. Hence we have

$$
\operatorname{gr} \tilde{K}(1)^{*}(B G) \cong \tilde{K}(1)^{*} \otimes\left(\mathbb{Z}\left[c_{18}, c_{24}\right]\left\{1,3 x_{4}\right\} \oplus E\right) .
$$

It is now unknown whether the element $x_{8}^{2} \in E$ is in the image of the cycle map. If it is so, then $g r_{g e o}^{*}(B G) \cong g r_{\text {top }}^{*}(B G)$, otherwise $g r_{g e o}^{i}(B G) ¥ g r_{\text {top }}^{i}(B G)$ for $i=12,16$.

\section{Rost MOtives}

In this section, we do not assume that $k$ is algebraically closed. At first, we recall the (generalized) Rost motive $([\mathrm{Ro} 1,2])$. Let $M(X)$ be the motive of (smooth) variety $X$. For a non zero symbol $a=\left\{a_{0}, \ldots, a_{n}\right\}$ in the $\bmod 2$ Milnor K-theory $K_{n+1}^{M}(k) / 2$, let $\phi_{a}=\left\langle\left\langle a_{0}, \ldots, a_{n}\right\rangle\right\rangle$ be the $(n+1)$-fold Pfister form. Let $X_{\phi_{a}}$ be the projective quadric of dimension $2^{n+1}-2$ defined by $\phi_{a}$. The Rost motive $M_{a}\left(=M_{\phi_{a}}\right)$ is a direct summand of the motive $M\left(X_{\phi_{a}}\right)$ representing $X_{\phi_{a}}$ so that $M\left(X_{\phi_{a}}\right) \cong M_{a} \otimes M\left(\mathbb{P}^{2^{n}-1}\right)$.

Moreover for an odd prime $p$ and nonzero symbol $0 \neq a \in K_{n+1}^{M} / p$, we can define ([Ro2],[Vo4,5],[Su-Jo]) the generalized Rost motive $M_{a}$, which is irreducible and is split over $K / k$ if and only if $\left.a\right|_{K}=0$ (as the case $p=2$ ).

The Chow group of the Rost motive is well known. Let $\bar{k}$ be an algebraic closure of $k,\left.X\right|_{\bar{k}}=X \otimes_{k} \bar{k}$, and $i_{\bar{k}}: C H^{*}(X) \rightarrow C H^{*}\left(\left.X\right|_{\bar{k}}\right)$ the restriction map. 
Lemma 7.1. (Rost [Ro1,2],[Vo4], [Vi-Ya], [Ya3,4]) The Chow group $C H^{*}\left(M_{a}\right)$ is only dependent on $n$. There are isomorphisms

$$
\begin{gathered}
C H^{*}\left(M_{a}\right) \cong \mathbb{Z}\{1\} \oplus\left(\mathbb{Z}\left\{c_{0}\right\} \oplus \mathbb{Z} / p\left\{c_{1}, \ldots, c_{n-1}\right\}\right)[y] /\left(c_{i} y^{p-1}\right) \\
\text { and } C H^{*}\left(\left.M_{a}\right|_{\bar{k}}\right) \cong \mathbb{Z}[y] /\left(y^{p}\right)
\end{gathered}
$$

where $2 \operatorname{deg}(y)=|y|=2\left(p^{n-1}+\ldots+p+1\right)$ and $\left|c_{i}\right|=|y|+2-2 p^{i}$. Moreover the restriction map is given by $i_{\bar{k}}\left(c_{0}\right)=$ py and $i_{\bar{k}}\left(c_{i}\right)=0$ for $i>0$.

Remark. The element $y$ does not exist in $C H^{*}\left(M_{a}\right)$ while $c_{i} y$ exists. Usually $C H^{*}\left(M_{a}\right)$ is defined only additively, however when $C H^{*}\left(M_{a}\right)$ has the natural ring structure (e.g., $p=2$ ), the multiplications are given by $c_{i} \cdot c_{j}=0$ for all $0 \leq i, j \leq$ $n-1$.

For the simplicity of notation, hereafter we always write by $\Omega^{*}(X)$ the $B P^{*}$ version of the algebraic cobordism

$$
\Omega^{*}(X) \otimes_{M U^{*}} B P^{*} \cong A B P^{2 * * *}(X) .
$$

Hence we mean $\Omega^{*}=B P^{*}$.

Let $I_{n}$ be the ideal in $\Omega^{*}$ generated by $v_{0}, \ldots, v_{n-1}$, i.e.,

$$
I_{n}=\left(p=v_{0}, v_{1}, \ldots, v_{n-1}\right) \subset \Omega^{*} .
$$

Then it is well known that $I_{n}$ and $I_{\infty}$ are the only prime ideals stable under the Landweber-Novikov cohomology operations ([Ra]) in $\Omega^{*}$.

The category of cobordism motives is defined and studied in [Vi-Ya]. In particular, we can define the algebraic cobordism of motives. The following is the main result in [Vi-Ya] (in [Ya4] for odd primes).

Lemma 7.2. ([Vi-Ya], [Ya4]) The restriction map

$$
i_{\bar{k}}: \Omega^{*}\left(M_{a}\right) \rightarrow \Omega^{*}\left(\left.M_{a}\right|_{\bar{k}}\right) \cong \Omega^{*}[y] /\left(y^{p}\right)
$$

is injective and there is an $\Omega^{*}$-module isomorphism

$$
\Omega^{*}\left(M_{a}\right) \cong \Omega^{*}\{1\} \oplus I_{n}\left\{y, \ldots, y^{p-1}\right\} \subset \Omega^{*}[y] /\left(y^{p}\right)
$$

such that $v_{i} y=c_{i}$ in $\Omega^{*}\left(M_{a}\right) \otimes_{\Omega^{*}} \mathbb{Z} \cong C H^{*}\left(M_{a}\right)$.

We consider the following assumption for $X$

Assumption (*). There is an isomorphism of motives

$$
M(X) \cong M_{n} \otimes A(X) \text { with } A(X) \cong \oplus_{s} \mathbb{T}^{i_{s}}
$$

where $\mathbb{T}$ is the $k$-Tate module.

Lemma 7.3. Suppose Assumption (*). Then

$$
K_{\text {alg }}^{0}(X) \cong K_{\text {alg }}^{0}\left(\left.X\right|_{\bar{k}}\right) \cong K_{\text {top }}^{0}(X(\mathbb{C})) .
$$

Proof. Since $M\left(\left.X\right|_{\bar{k}}\right)$ is a sum of $\bar{k}$-Tate modules, we have the isomorphism $K_{\text {alg }}^{0}\left(\left.X\right|_{\bar{k}}\right) \cong$ $K_{\text {top }}^{0}(X(\mathbb{C}))$ from

$$
K_{\text {alg }}^{0}(\mathbb{T}) \cong K_{\text {alg }}^{0}\left(\left.S^{2,1}\right|_{\bar{k}}\right) \cong K_{\text {top }}^{0}\left(S^{2}\right) .
$$

For the first isomorphism, we only need to show $K_{\text {alg }}^{0}\left(M_{n}\right) \cong K_{\text {alg }}^{0}\left(\left.M_{n}\right|_{\bar{k}}\right)$. Recall

$$
\Omega^{*}\left(M_{n}\right) \cong\left(B P^{*} \oplus \operatorname{Ideal}\left(p, v_{1}, \ldots, v_{n-1}\right)\{\rho\}\right)
$$

by $c_{i} \mapsto v_{i} \rho$. Hence $v_{i} c_{1}=v_{1} c_{i}$. Therefore for $i>1$, we see $c_{i}=0$ in $A \tilde{K}(1)^{2 *, *}\left(M_{n}\right)$ where $v_{i}=0$. So we have

$$
A \tilde{K}(1)^{2 * * *}\left(M_{n}\right) \cong \tilde{K}(1)^{*}\left\{1, c_{0}, c_{1}\right\} /\left(v_{1} c_{0}=p c_{1}\right) \cong A \tilde{K}(1)^{2 *, *}\left\{1, c_{1}\right\}
$$




$$
\cong A \tilde{K}(1)^{2 * *}\left\{1, v_{1} \rho\right\} \cong A \tilde{K}(1)^{2 *, *}\{1, \rho\} \cong A \tilde{K}(1)^{2 *, *}\left(\left.M\right|_{\bar{k}}\right) .
$$

\section{FLAG MANIFOLDS $G / T$}

Now we consider the flag variety $G / T$. Let $G$ be a simply connected Lie group and $T$ the maximal torus. Moreover we assume its cohomology is

$$
H^{*}(G ; \mathbb{Z} / p) \cong \mathbb{Z} / p[y] /\left(y^{p}\right) \otimes \Lambda\left(x_{1}, \ldots, x_{\ell}\right)
$$

with $|y|=2(p+1)$ and $\left|x_{i}\right|=o d d$. Then it is well known that the cohomology of $G / T$ is torsion free ([Tod]) and

$$
H^{*}(G / T) \cong \mathbb{Z}\left[y, t_{1}, \ldots, t_{\ell}\right] /\left(f_{y}, b_{1}, \ldots, b_{\ell}\right)
$$

where $f_{y}=y^{p} \bmod \operatorname{Ideal}\left(t_{i}\right)$ and $\left(b_{1}, \ldots, b_{\ell}\right)$ is a regular sequence in $\mathbb{Z}\left[t_{1}, \ldots, t_{\ell}\right]$.

Let $k$ be a subfield of $\mathbb{C}$ which contains primitive $p$-th root of the unity. Let us denote by $G_{k}$ the split reductive group over $k$ which corresponds $G$. By definition, a $G_{k}$-torsor $\mathbb{G}_{k}$ over $k$ is a variety over $k$ with a free $G_{k}$-action such that the quotient variety is $\operatorname{Spec}(k)$. A $G_{k}$-torsor over $k$ is called trivial, if it is isomorphic to $G_{k}$ or equivalently it has a $k$-rational point. In this paper by $\mathbb{G}_{k}$, we mean the nontrivial torsor at any finite extension $K / k$ coprime to $p$.

Let $H$ be a subgroup of $G$. Given a torsor $\mathbb{G}_{k}$ over $k$, we can form the twisted form of $G / H$ by

$$
\left(\mathbb{G}_{k} \times G_{k} / H_{k}\right) / G_{k} \cong \mathbb{G}_{k} / H_{k}
$$

Letting $X=G / T$, we consider cases such that Assumption hold. By [Pe-Se-Za], exceptional Lie groups $\left(G_{2}, p=2\right)$ and $\left(F_{4}, p=3\right)$ are such cases. The filtrations of such spaces are also studied by Gabrier and Zainouline ([Ga-Za], [Za], [Ju]) as the twisted gamma filtrations.

At first, we consider the case $(G, p)=\left(G_{2}, 2\right)$. We recall the cohomology from Toda-Watanabe [To-Wa]

$$
H^{*}(G / T ; \mathbb{Z}) \cong \mathbb{Z}\left[t_{1}, t_{2}, y\right] /\left(t_{1}^{2}+t_{1} t_{2}+t_{2}^{2}, t_{2}^{3}-2 y, y^{2}\right)
$$

with $\left|t_{i}\right|=2$ and $|y|=6$. Let $P$ be the maximal parabolic subgroup such that $G / P$ is isomorphic to a quadric. Then from (3.6) and $H^{*}(P / T) \cong \mathbb{Z}\left\{1, t_{1}\right\}$ ([To-Wa])

$$
H^{*}(G / P ; \mathbb{Z}) \cong \mathbb{Z}\left[t_{2}, y\right] /\left(t_{2}^{3}-2 y, y^{2}\right) \cong \mathbb{Z}\{1, y\} \otimes\left\{1, t_{2}, t_{2}^{2}\right\}
$$

Of course this is isomorphic to $g r_{\text {top }}^{*}(G / P)$.

Since $G / P$ is a quadric, we have the decomposition ([Bo], $\S 7$ in [Pe-Se-Za])

$$
M\left(\mathbb{G}_{k} / P_{k}\right) \cong M_{2} \oplus M_{2}(1) \oplus M_{2}(2) .
$$

Theorem 8.1. (Theorem 5.2 in[Ya5]) There is a ring isomorphism

$$
\begin{gathered}
g r_{\gamma}^{*}(G / P) \cong g r_{\text {geo }}^{*}\left(\mathbb{G}_{k} / P_{k}\right) \cong C H^{*}\left(\mathbb{G}_{k} / P_{k}\right) \\
\cong \mathbb{Z}_{(2)}\left[t_{2}, u\right] /\left(t_{2}^{6}, 2 u, t_{2}^{3} u, u^{2}\right) \cong \mathbb{Z}_{(2)}\left[t_{2}\right] /\left(t_{2}^{6}\right) \oplus \mathbb{Z} / 2\left[t_{2}\right] /\left(t_{2}^{3}\right)\{u\}
\end{gathered}
$$

with $\left|t_{2}\right|=2,|u|=4$.

Proof. Recall that

$$
\Omega^{*}\left(M_{2}\right) \cong \Omega^{*}\{1,2 y, v y\} \subset \Omega^{*}\{1, y\} .
$$

From the decomposition of the motive, we have the $\Omega^{*}$-module isomorphism

$$
\Omega^{*}\left(\mathbb{G}_{k} / P_{k}\right) \cong \Omega^{*}\left\{1, v_{1} y, 2 y\right\} \otimes\left\{1, t_{2}, t_{2}^{2}\right\} \subset \Omega^{*}\left(G_{k} / P_{k}\right)
$$


Since $C H^{*}(X) \cong \Omega^{*}(X) \otimes_{\Omega^{*}} \mathbb{Z}$, we have the isomorphism

$$
C H^{*}\left(\mathbb{G}_{k} / P_{k}\right) \cong \mathbb{Z}\{1,2 y\}\left\{1, t_{2}, t_{2}^{2}\right\} \oplus \mathbb{Z} / 2\left\{v_{1} y\right\}\left\{1, t_{2}, t_{2}^{2}\right\} .
$$

(Note $2 v_{1} y=v_{1}(2 y) \in \Omega^{<0} \Omega^{*}\left(\mathbb{G}_{k} / P_{k}\right)$.)

Here the multiplications are given as follows. Since $2 y=t_{2}^{3} \bmod \left(\Omega^{<0}\right)$ in $\Omega^{*}\left(G_{k} / T_{k}\right)$, we can take $2 y=t_{2}^{3} \in C H^{*}\left(\mathbb{G} / P_{k}\right)$ so that

$$
\mathbb{Z}\{1,2 y\}\left\{1, t_{2}, t_{2}^{2}\right\}=\mathbb{Z}\left[t_{2}\right] /\left(t_{2}^{6}\right) \subset C H^{*}\left(\mathbb{G} / P_{k}\right) .
$$

Let us write $u=v_{1} y$ in $C H^{*}\left(\mathbb{G}_{k} / T_{k}\right)$. Then $t_{2}^{3} u=2 y v_{1} y=0$ and $u^{2}=v_{1}^{2} y^{2}=0$ in $\Omega^{*}\left(\mathbb{G}_{k} / T_{k}\right) \otimes_{\Omega^{*}} \mathbb{Z}$. Hence we have the second isomorphism for in the theorem.

Since $|u|=4$, the element $u$ is represented by Chern classes, we see the first isomorphism.

Remark. The space $\mathbb{G}_{k} / T_{k}$ is isomorphic to the quadric defined by the maximal neighbor of the 3-Pfister form. Hence its Chow ring is computed in [Ya5].

It is well known that the representations (over $\mathbb{C})$ ) are written as

$$
R(G / T) \cong R(T) / R(G) .
$$

Therefore each element which is represented by Chern classes is written as an element in $\Omega^{*}\left(\mathbb{G}_{k} / T_{k}\right)$

$$
c(\xi)=\prod\left(1+\lambda_{1} t_{1}+\lambda_{2} t_{2}\right) \in \Omega^{*}\left[t_{1}, t_{2}\right] \quad \lambda_{i} \in \mathbb{Z} / 2
$$

modulo $\left(\left(t_{1}, t_{2}\right) \Omega^{<0} \Omega^{*}\left(G_{k} / T_{k}\right)\right)$. By the similar arguments, we have (see Theorem 5.3 in $[$ Ya5])

Theorem 8.2. There are ring isomorphisms

$$
g r_{\gamma}^{*}(G / T) \cong C H^{*}\left(\mathbb{G}_{k} / T_{k}\right) \cong \mathbb{Z}\left[t_{1}, t_{2}\right] /\left(t_{2}^{6}, 2 u, t_{2}^{3} u, u^{2}\right)
$$

where $u=t_{1}^{2}+t_{1} t_{2}+t_{2}^{2}$.

Proof. The Chow ring is isomorphic to

$$
\begin{aligned}
& (*) \quad C H^{*}\left(\mathbb{G}_{k} / T_{k}\right) \cong C H^{*}\left(\mathbb{G}_{k} / P_{k}\right)\left\{1, t_{1}\right\} \\
& \cong\left(\mathbb{Z}\{1,2 y\} \oplus \mathbb{Z} / 2\left\{v_{1} y\right\}\right)\left\{1, t_{2}, t_{2}^{2}\right\}\left\{1, t_{1}\right\} .
\end{aligned}
$$

Here $2 y=t_{2}^{3}$. Since $v_{1} y \in\left(t_{1}, t_{2}\right)$ and $v_{1} y=0 \in C H^{*}\left(G_{k} / T_{k}\right)$, we see

$$
v_{1} y=\lambda\left(t_{1}^{2}+t_{1} t_{2}+t_{2}^{2}\right) \quad \bmod \left(\left(t_{1}, t_{2}\right) \Omega^{<0} \Omega^{*}\left(G_{k} / T_{k}\right)\right)
$$

for $\lambda \in \mathbb{Z}$. We can take $\lambda=1 \bmod (2)$. Otherwise $v_{1} y=0 \in \Omega^{*}\left(G_{k} / T_{k}\right) / 2$, which is an $\Omega^{*} / 2$-free, and this is a contradiction. Hence we can take $t_{1}^{2}+t_{1} t_{2}+t_{2}^{2}$ as $v_{1} y$. Hence in $C H^{*}\left(\mathbb{G}_{k} / T_{k}\right)$ we have the relation

$$
\left(t_{2}^{3}\right)^{2}=0,\left(t_{2}^{3}\right) u=0, u^{2}=0,2 u=0 .
$$

Next we consider the case $(G, p)=\left(F_{4}, 3\right)$. Let $\mathbb{G}_{k}$ be a nontrivial $G_{k}$-torsor at 3 as previous sections. Let $P_{k}$ be a maximal parabolic subgroup of $G_{k}$ given by the the first three vertexes

$$
\stackrel{1}{\circ}--\stackrel{2}{\circ} \Rightarrow=\stackrel{3}{\circ}--\stackrel{4}{\circ}
$$

of the Dynkin diagram. Then Nikolenko- Semenov-Zainoulline ([Ni-Se-Za]) showed that there is an isomorphism

$$
M\left(\mathbb{G}_{k} / P_{k}\right) \cong \oplus_{i=0}^{7} M_{2}(i) .
$$


We first recall the ordinary cohomology of $G / P([\mathrm{Is}-\mathrm{To}],[\mathrm{Du}-\mathrm{Za}])$.

$$
H^{*}(G / P)_{(3)} \cong \mathbb{Z}[t, y] /\left(r_{8}, r_{12}\right), \quad|t|=2,|y|=8
$$

where $r_{8}=3 y^{2}-t^{8}$ and $r_{12}=26 y^{3}-5 t^{12}$. Hence we can rewrite

$$
H^{*}(G / P) \cong \mathbb{Z}\left\{1, t, \ldots, t^{7}\right\} \otimes\left\{1, y, y^{2}\right\} .
$$

Recall the Rost motive $C H^{*}\left(\left.M_{2}\right|_{\bar{k}}\right) \cong \mathbb{Z}[y] /\left(y^{3}\right)$,

$$
C H^{*}\left(M_{2}\right) \cong \mathbb{Z}\{1\} \oplus \mathbb{Z}\left\{3 y, 3 y^{2}\right\} \oplus \mathbb{Z} / 3\left\{v_{1} y, v_{1} y^{2}\right\} .
$$

Of course, the above $y \in C H^{*}\left(M_{a}\right)$ can be identified with the same named element in $H^{*}\left(G_{k} / P_{k}\right)$ by the restriction map $C H^{*}\left(M_{a}\right) \rightarrow C H^{*}\left(\left.M_{a}\right|_{\bar{k}}\right) \subset C H^{*}\left(G_{k} / P_{k}\right)$. From the above theorem, we have the decomposition

$$
(*) C H^{*}\left(\mathbb{G}_{k} / P_{k}\right) \cong \mathbb{Z}\left\{1, t, \ldots, t^{7}\right\} \otimes\left(\mathbb{Z}\left\{1,3 y, 3 y^{2}\right\} \oplus \mathbb{Z} / 3\left\{v_{1} y, v_{1} y^{2}\right\}\right) .
$$

The ring structure is given as follows.

Proposition 8.3. ( Theorem 6.2 in [Ya5])

$$
\begin{gathered}
g r_{\text {geo }}^{*}\left(\mathbb{G}_{k} / P_{k}\right) \cong C H^{*}\left(\mathbb{G}_{k} / P_{k}\right) \\
\cong \mathbb{Z}\left[t, b, a_{1}, a_{2}\right] /\left(t^{16}, t^{8} b, b^{2}=3 t^{8}, b a_{i}, 3 a_{i}, t^{8} a_{i}, a_{1} a_{2}\right) \\
\cong \mathbb{Z}\left\{1, t, \ldots, t^{7}\right\} \otimes\left(\mathbb{Z}\left\{1, \sqrt{ } 3 t^{4}, t^{8}\right\} \oplus \mathbb{Z} / 3\left\{a_{1}, a_{2}\right\}\right)
\end{gathered}
$$

where $|b|=8$ and $\left|a_{1}\right|=4,\left|a_{2}\right|=12$.

Proof. From the relation $r_{8}$ in $C H^{*}(G / P)$, we have

$$
3 y^{2}=t^{8}+v x \in \Omega^{*}(G / P) \quad \text { for } v \in \Omega^{<0} .
$$

Hence we can take $t^{8}$ instead of $3 y^{2}$ in $(*)$. Of course

$$
(3 y)^{2}=3 t^{8}+3 v x \in \Omega^{*}\left(G_{k} / P_{k}\right) .
$$

Hence we write by $b=\sqrt{ } 3 t^{4}$ the element $3 y$. Write by $a_{1}, a_{2}$ the elements $v_{1} y, v_{1} y^{2}$ respectively. Elements in $I_{\infty} \Omega^{<0} \subset \Omega\left(G_{k} / P_{k}\right)$ reduces to zero in $C H^{*}\left(\mathbb{G}_{k} / T_{k}\right)$. Therefore we have the desired multiplicative results.

The element $b=3 y$ is represented by a Chern class $c_{4}(\xi)$ for some $\xi$ by the Riemann-Roch theorem without denominators. Unfortunately, we do not know if $a_{2}=v_{1} y^{2}$ are Chern classes in $C H^{*}(B G)$ or not.

Proposition 8.4. If $a_{2}=v_{1} y^{2} \in C H^{*}\left(\mathbb{G}_{k} / P_{k}\right)$ is not represented by a Chern class, then

$$
g r_{\gamma}(G / P) \cong \mathbb{Z}\left[t, b, a_{1}\right] /\left(t^{16}, t^{8} b, b^{2}=3 t^{8}, b a_{1}, 3 a_{1}, t^{8} a_{1}, a_{1}^{3}\right)
$$

where $|b|=8$ and $\left|a_{1}\right|=4$.

Proof. If $v_{1} y^{2}$ is not represented by Chern class of $C H^{*}\left(\mathbb{G}_{k} / P k\right)\left(\right.$ or $\left.\Omega^{*}\left(\mathbb{G}_{k} / P_{k}\right)\right)$, then the corresponding nonzero element in $g r_{\gamma}(G / T)$ is $v_{1}^{2} y^{2}$, which is written as $\left(v_{1} y\right)^{2}=\left(a_{1}\right)^{2}$. 


\section{Filtrations of the Morava $K$-Theory}

For most groups $G$ in the preceding sections, it is known that $K(n)^{\text {odd }}(B G)=0$ (while Kriz gave some examples with $K(n)^{\text {odd }}(B G) \neq 0$ ). Hereafter, we only consider spaces $X$ such that

$$
\begin{gathered}
\text { (9.1) } \quad K(n)^{\text {odd }}(X(\mathbb{C}))=\tilde{K}(n)^{\text {odd }}(X(\mathbb{C}))=0 \\
(9.2) \quad K(n)^{*}(X(\mathbb{C})) \cong A K(n)^{2 *, *}(X)
\end{gathered}
$$

Then we can define the three filtrations for the Morava $K(n)$-theory.

$$
\begin{gathered}
F(n)_{\text {top }}^{2 i}=K \operatorname{Ker}\left(K ( n ) ^ { * } \left(X(\mathbb{C}) \rightarrow K(n)^{*}\left(X(\mathbb{C})^{2 i}\right),\right.\right. \\
F(n)_{g e o}^{2 i}=\left\{f_{*}\left(1_{M}\right) \mid f: M \rightarrow X \text { and } \operatorname{codim}_{X} M \geq i\right\} \\
F(n)_{\gamma}^{2 i}=\left\{c_{i_{1}}^{K(n)}\left(x_{1}\right) \cdot \ldots \cdot c_{i_{m}}^{K(n)}\left(x_{m}\right) \mid i_{1}+\ldots i_{m} \geq i\right\} .
\end{gathered}
$$

Here $c_{i_{s}}^{K(n)}\left(x_{s}\right)$ is the Chern class for $A K(n)^{*, *^{\prime}}$-theory for some $k$-representation

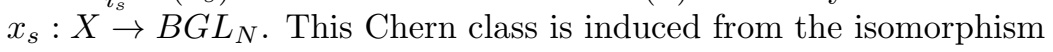

$$
A K(n)^{2 * * *}\left(B G L_{N}\right) \cong K(n)^{*} \otimes_{B P^{*}} \Omega^{*}\left(B G L_{N}\right),
$$

in fact, it is well known that in $\Omega^{*}(X)$, we can define Chern classes canonically (see [Mo-Le] for example). However each element in $K(n)^{*}(X(\mathbb{C}))$ (for $n \geq 2$ ) need not to be represented by $K(n)^{*}$-theory Chern classes. Hence we need the assumption

$$
\text { (9.3) } \quad F_{\gamma}^{0}=K(n)^{*}(X) \text {. }
$$

(We also consider the cases where (9.3) does not hold.) Of course the assumptions are satisfied for $K(1)^{*}$-theory, if they are so for $\tilde{K}(1)^{*}$-theory.

Recall $P(n)^{*}(X)$ be the cohomology theory with the coefficient

$$
P(n)^{*}=B P^{*} /\left(p, v_{1}, \ldots, v_{n-1}\right) .
$$

It is well known, for all $X$,

$$
P(n)^{*}(X) \otimes_{B P^{*}} K(n)^{*} \cong K(n)^{*}(X) .
$$

Let us write by $E(P(n))_{r}^{*, *^{\prime}}$ (resp. $E(K(n))_{r}^{*, *^{\prime}}$ ) the AHss converging to $P(n)^{*}(X)$ (resp. $\left.K(n)^{*}(X)\right)$. Then we have

$$
E(P(n))_{r}^{*, *^{\prime}} \otimes_{B P^{*}} K(n)^{*} \cong E(K(n))_{r}^{*, *^{\prime}} .
$$

If (9.1)-(9.3) are satisfied, then $K(n)$-version (exchanging $B P^{*}(X)$ to $\left.P(n)^{*}(X)\right)$. of all lemmas in $\S 2$ also hold.

Lemma 9.1. Suppose that $\Omega^{*}(X) / p \cong B P^{*}(X(\mathbb{C})) / p$ and it is generated by $\left(B P^{*}\right.$ ) Chern classes. Then (9.1)-(9,3) are satisfied.

Proof. We consider the maps

$$
\Omega^{*}(X) \otimes_{B P^{*}} K(n)^{*} \stackrel{\rho_{1}}{\rightarrow} A K^{2 *, *}(X) \stackrel{\rho_{2}}{\rightarrow} K(n)^{*}(X(\mathbb{C})) .
$$

Here the map $\rho_{1}$ is an epimorphism because $\Omega^{*}(X)$ (resp. $\left.A K(n)^{2 * *}(X)\right)$ is generated as a $B P^{*}$-module (resp. $K(n)^{*}$-module) by elements in $C H^{*}(X)$.

On the other hand by Ravenel-Wilson-Yagita [Ra-Wi-Ya], we know that (1.2) implies

$$
K(n)^{*}(X(\mathbb{C})) \cong K(n)^{*} \otimes_{B P^{*}} B P^{*}(X(\mathbb{C})) .
$$

From the supposion in the theorem, we see that $\rho_{2} \rho_{1}$ is an isomorphism. This means that $\rho_{1}, \rho_{2}$ are also isomorphisms. 
For $X=B G, G=$ finite abelian, $p_{ \pm}^{1+2}, O_{n}, G_{2}$ and $P S L_{3}(p=3)$ satisfy the assumptions in the above lemma.

Of course $g r_{\text {top }}^{*}(X)$ and $g r(n)_{\text {top }}^{*}(X)$ are quite different. Let $G=\mathbb{Z} / p$. Then

$$
K(n)^{*}(B G) \cong K(n)^{*}(y] /\left(y^{p^{n}}\right) .
$$

and this is generated by Chern classes in $H^{*}(B G ; \mathbb{Z} / p)$.

Theorem 9.2. Let $G=\oplus^{s} \mathbb{Z} / p$. Then all three filtrations of $K(n)^{*}(B G)$ are same and

$$
g r(n)_{\text {top }}^{*}(B G) \cong \mathbb{Z} / p\left[y_{1}, \ldots, y_{s}\right] /\left(y_{1}^{p^{n}}, \ldots, y_{s}^{p^{n}}\right) .
$$

Similarly, we have

Theorem 9.3. Let $G=O_{m}$. Then all three filtrations of $K(n)^{*}(B G)$ are same and

$$
\operatorname{gr}(n)_{\text {top }}^{*}(B G) \cong\left\{\sum y_{1}^{i_{1}} \ldots y_{s}^{i_{s}}\left(y_{s+1} y_{s+2}\right)^{j_{s+1}} \ldots\left(y_{2 k+1} y_{2 k+2}\right)^{j_{2 k+1}}\right\}
$$

where $0 \leq i_{1} \leq \ldots \leq i_{s}<2^{n} \leq i_{s} \leq \ldots \leq i_{k}$.

For example, $g r(n)_{\text {top }}^{*} \cong \mathbb{Z} / 2\left[c_{2}\right] \oplus \mathbb{Z} / 2\left\{c_{1}^{i} c_{2}^{j} \mid i+2 j<2^{n}\right\}$.

Next we consider the case $G=S O_{2 m}$ Recall for $m \geq 3, y_{2 m}$ is not represented by Chern classes

Theorem 9.4. Let $G=S O_{2 m}$ and $m>2$. Then

$$
\operatorname{gr}(n)_{\text {geo }}^{*}(B G) \cong \mathbb{Z}\left[c_{2}, c_{4}, \ldots, c_{2 m}\right]\left\{y_{2 m}\right\} \oplus \operatorname{gr}(n)_{g e o}^{*}\left(B O_{2 m}\right) /\left(c_{1}\right) .
$$

However $\operatorname{gr}(n)_{\gamma}^{*}(B G) \nRightarrow g r(n)_{\text {geo }}^{*}(B G) \nRightarrow g r(n)_{\text {top }}^{*}(B G)$.

Proof. We only need the second nonisomorphism of the second statement. Since $y_{2 m}=(-1)^{*} 2^{m-1} w_{2 m} \in H^{*}(B G)$ is zero in $H^{*}(B G ; \mathbb{Z} / 2)$. Hence $0 \neq y_{2 m} \in$ $P(n)^{*}(B G)$ is represented in the AHss converging to $P(n)^{*}(B G)$ as element in $E_{\infty}^{*, *^{\prime}}$ with $*^{\prime}<0$ and $*>2 m$.

Next consider the case $G=G_{2}$ (and $p=2$ ). By the computation of the AHss for $P(1)^{*}(B G)\left(=B P^{*}(B G ; \mathbb{Z} / 2)\right)$, we have

$$
K(1)^{*}(B G) \cong K(1)^{*}\left[c_{4}, c_{6}\right]\left\{1, v_{1} w_{6}\right\} .
$$

By the direct computation of the AHss for $K(2)^{*}(B G)$, we see

$$
K(2)^{*}(B G) \cong K(1)^{*}\left[c_{4}, c_{6}\right]\left\{1, w_{4} w_{6}\right\} .
$$

Therefore we have

Theorem 9.5. Let $G=G_{2}$. Then

$$
\begin{aligned}
& \operatorname{gr}(i)_{\alpha}^{*}(B G) \cong \mathbb{Z} / 2\left[c_{4}, c_{6}\right]\{1, a\} \\
& \text { where } a^{2}=\left\{\begin{array}{l}
c_{4} c_{6}|a|=10 \quad \text { if } i=2 . \alpha=\text { top } \\
c_{6}|a|=6 \quad \text { if } i=1 . \alpha=\text { top } \\
0|a|=4 \quad \text { if } i=1,2 . \alpha \neq \text { top. }
\end{array}\right. \text {. }
\end{aligned}
$$

Proof. The above $a$ is represented as $a=w_{4} w_{6}$ (resp. $\left.w_{6}, v_{1} w_{6}, v_{2} w_{4} w_{6}\right)$ when $i=2, \alpha=\operatorname{top}($ resp. $i=1, \alpha=t o p, i=1 \alpha \neq t o p$ ), and $i=2 \alpha \neq t o p)$ ).

When $n \geq 1$, the geometric and topological filtrations are quite different. 
Theorem 9.6. Let $G$ be a simply connected simple Lie group such that $H^{*}(G)$ has p-torsion. Then for $n \geq 1$

$$
\operatorname{gr}(n)_{g e o}^{4}(B G) \neq 0 \quad \text { but } \quad \operatorname{gr}(n)_{\text {top }}^{4}(B G)=0 .
$$

Proof. The space $B G$ is 3 -connected and $H^{4}(B G) \cong \mathbb{Z}$. Let us write by $x$ its 4 dimensional generator. By [Ka-Ya2], it is known that $p x \in H^{4}(B G)$ is represented as the Chern class $c_{2}$ for some representation. Hence $\operatorname{gr}(n)_{g e o}^{4}(B G) \neq 0$.

To see $g r(n)_{\text {top }}^{4}(B G)=0$, we only need to show

$$
\text { (*) } \quad d_{2 p^{n}-1}(x)=v_{n} \otimes Q_{n}(x) \neq 0
$$

in the AHss converging to $K(n)^{*}(B G)$.

For these groups, it is well known that there are embedding $G_{2} \subset G$ for $p=2$, $\left(F_{4} \subset G\right.$ for $p=3$ and $G=E_{8}$ for $\left.p=5\right)$. We will prove $(*)$ for $G=F_{4}$ and $p=3$, then we can see $(*)$ the other groups when $p=3$. (The other primes cases are similar).

Let $G=F_{4}$ and $p=3$. Then $G$ has a maximal elementary $p$-group $A \cong(\mathbb{Z} / 3)^{3}$. We consider the restriction map for $i: A \subset G$,

$$
i^{*}: H^{*}(B G ; \mathbb{Z} / p) \rightarrow H^{*}(B A ; \mathbb{Z} / p) \cong \mathbb{Z} / p\left[y_{1}, y_{2}, y_{3}\right] \otimes \Lambda\left(x_{1}, x_{2}, x_{3}\right)
$$

The restriction image is $i^{*}(x)=Q_{0}\left(x_{1} x_{2} x_{3}\right)$ (see [Ka-Te-Ya]). Hence we show

$$
i^{*}\left(Q_{n}(x)\right)=Q_{n} Q_{0}\left(x_{1} x_{2} x_{3}\right)=\sum y_{1}^{p^{n}} y_{2} x_{3} \neq 0 \text {. }
$$

Now we recall arguments for quadrics. Let $m=2 m^{\prime}+1$. and let us write the quadratic form $q(x)$ defined by

$$
q\left(x_{1}, \ldots, x_{m}\right)=x_{1} x_{2}+\ldots+x_{m-2} x_{m-1}+x_{m}^{2}
$$

and the projective quadric $X_{q}$ defined by the quadratic form $q$. Then it is well known that (in fact $S O(m)$ acts on the affine quadric in $\mathbb{A}^{m}-0$ )

$$
X_{q} \cong S O(m) /(S O(m-2) \times S O(2)) .
$$

Let $G=S O(m)$ and $P=S O(m-2) \times S O(2)$. Then the quadric $q$ is always split over $k$ and we know $C H^{*}\left(G_{k} / P_{k}\right) \cong C H^{*}\left(X_{q}\right)$.

In particular we consider the case $m=2^{n+1}-1$. Let $\rho=\{-1\} \in K_{1}^{M}(k) / 2=$ $k^{*} /\left(k^{*}\right)^{2}$. We consider fields $k$ such that

$$
0 \neq \rho^{n+1} \in K_{n+1}^{M}(k) / 2 .
$$

Define the quadratic form $q^{\prime}$ by

$$
q^{\prime}\left(x_{1}, \ldots, x_{m}\right)=x_{1}^{2}+\ldots+x_{m}^{2} .
$$

Then this $q^{\prime}$ is a subform of

$$
\langle\langle-1, \ldots,-1\rangle\rangle=\phi_{\rho^{n+1}}
$$

the $(n+1)$-th Pfister form associated to $\rho^{n+1}$. (That is, $q^{\prime}$ is the maximal neighbor of the $(n+1)$-th Pfister form.) Of course $\left.q\right|_{\bar{k}}=\left.q^{\prime}\right|_{\bar{k}}$ and we can identify $\mathbb{G}_{k} / P_{k} \cong$ $X_{q^{\prime}}$. From Lemma 7.2 (or Rost's result), we know

$$
C H^{*}\left(\left.X_{q^{\prime}}\right|_{\bar{k}}\right) \cong \mathbb{Z}[t, y] /\left(t^{2^{n}-1}-2 y, y^{2}\right) .
$$

As stated in $\S 7$, there is a decomposition of motives

$$
M\left(X_{q^{\prime}}\right) \cong M_{n} \otimes \mathbb{Z} / 2[t] /\left(t^{2^{n}-1}\right) .
$$


Hence we have the additive isomorphism

$$
C H^{*}\left(X_{\phi_{a}^{\prime}}\right) \cong \mathbb{Z}[t] /\left(t^{2^{n}-1}\right) \otimes\left(\mathbb{Z}\left\{1, c_{n, 0}\right\} \oplus \mathbb{Z} / 2\left\{c_{n, 1}, \ldots, c_{n, n-1}\right\}\right) .
$$

With identification $t^{2^{n}-1}=2 y=c_{n, 0}$, and $u_{i}=c_{n, i}$ for $i>0$, we also get the ring isomorphism

Theorem 9.7. ([Ya5]) Let $G_{k} / P_{k}$ be the above quadric $X_{q^{\prime}}$. Then there is a ring isomorphism

$$
C H^{*}\left(\mathbb{G}_{k} / P_{k}\right) \cong \mathbb{Z}[t] /\left(t^{2^{n+1}-2}\right) \oplus \mathbb{Z} / 2[t] /\left(t^{2^{n}-1}\right)\left\{u_{1}, \ldots, u_{n-1}\right\}
$$

where $u_{i}=v_{i} y \in \Omega^{*}\left(\mathbb{G}_{k} / p\right) \otimes_{\Omega^{*}} Z_{(2)}$ so $u_{i} u_{j}=0$. Hence we have for $1 \leq i \leq n-1$

$$
\operatorname{gr}(i)_{\text {geo }}\left(\mathbb{G}_{k} / P_{k}\right) \cong \mathbb{Z}[t] /\left(t^{2^{n+1}-2}\right) \oplus \mathbb{Z} / 2[t] /\left(t^{2^{n}-1}\right)\left\{u_{i}\right\} .
$$

Proof. In $K(i)^{*}(X)$, we see $v_{j}=0$ for $i \neq j$. Since $v_{j} u_{i}=v_{i} u_{j}$, we see $u_{j}=0$ for $i \neq j$.

\section{REFERENCES}

[Ad] J. F. Adams. Lectures on exceptional Lie groups, Univ. Chicago Press, Chicago, IL, 1996.

[At] M. Atiyah. Chatracters and the cohomology of finite groups. Publ. Math. IHES, 9 (1961) 23-64.

[Bo] J.Bonnet. Un isomorphisme motivique entre deux variétés homogénes projective sous l'action d'un groupe de type $G_{2}$. Doc. Math. 8 (2003) 247-277.

[Ca-Pe-Se-Za] B.Calmès, V.Petrov, N.Semenov and K.Zainoulline. Chow motives of twisted flag varieties. Compositio Math. 142 (2006) 1063-1080.

[Du-Za] H.Duan and X.Zhao The Chow ring of generalized Grassmanianns. Found. Comput. Math. 10 (2010) 245-274.

[Ga-Me-Se] S.Garibaldi, A.Merkurjev, and J.P.Serre. Cohomological invariants in Galois cohomology. University lect. series vol(28) AMS (2003).

[Fi] R. Field. The Chow ring of the classifying space BSO(2n, C). J. Algebra 349 (2012) 330-339.

[Ga-Za] S. Garbardi and K.Zainoulline. The gamma filktration and the Rost invariant. to appear in J. Reine Angew. Math. Math. arXiv:1007. 3482 (2010) 19pp. .

[Gr] A. Grothendieck. Torsion homologique et sections rationnelles. Sem. C.Chevalley, ENS 1958, expose 5, Secreatariat Math., IHP, Paris (1958).

[Gu] P.Guillot. The Chow ring of $G_{2}$ and Spin(7). J. Reine angew. Math. 604 (2007) 137-158.

[Ha] M.Hazewinkel. Formal groups and applications. Pure and Applied Math. 78, Academic Press Inc. (1978) xxii+573pp.

[Is-To] K. Ishitoya, and H. Toda. On the cohomology of irreducible symmetric spaces of exceptional type. J.Math. Kyoto Univ. 17 (1977), 225-243.

[Ju] C. Junkins. The J-invariant and Tits algebras for groups of inner type $E_{6}$. manuscripta math. 140 (2013), 249-261.

[Ka-Ya1] M.Kameko and N.Yagita. The Brown-Peterson cohomology of the classifying spaces of the projective unitary groups $P U(p)$ and exceptional Lie group. Trans. Amer. Math. Soc. 360 (2008), 2265-2284.

[Ka-Ya2] M.Kameko and N.Yagita. Chern subrings. Proc. Amer. Math. Soc. 138 (2010) 367373.

[Ko-Ya] A. Kono and N. Yagita. Brown-Peterson and ordinary cohomology theories of classifying spaces for compact Lie groups, Trans. Amer. Math. Soc. 339 (1993), no. 2, 781-798.

[Le-Ya] I. Leary and N. Yagita. Some examples in the integral and Brown-Peterson cohomology of p-groups.. Bull. London Math. Soc. 24 (1992) 165-168.

[Le-Mo 1] M. Levine and F. Morel. Cobordisme algébrique I. C. R. Acad. Sci. Paris 332 (2001), 723-728. 
[Le-Mo 2] M. Levine and F. Morel. Cobordisme algébrique II. C. R. Acad. Sci. Paris 332 (2001), 815-820.

[Mo-Vi] L.Molina and A.Vistoli. On the Chow rings of classifying spaces for classical groups. Rend. Sem. Mat. Univ. Padova 116 (2006), 271-298.

[Ni-Se-Za] S. Nikolenko, N.Semenov and K.Zainoulline. Motivic decomposition of anisotropic varieties of type $F_{4}$ into generalized Rost motives. J. K-theory 3 (2009), 85-102.

[Pe-Se-Za] V.Petrov, N.Semenov and K.Zainoulline. J-invariant of linear algebraic groups. Ann. Sci. Ec. Norm Super. 41 (2008) 1023-1053.

[Qu] D. Quillen Themod 2 cohomology rings of extra-special 2-groups and the spinor groups. Math. Ann. 194 (1971) 197-212.

[Ra] D.Ravenel. Complex cobordism and stable homotopy groups of spheres. Pure and Applied Mathematics, 121. Academic Press (1986).

[Ro1] M.Rost. Some new results on Chowgroups of quadrics. preprint (1990).

[Ro2] M.Rost. On the basic correspondence of a splitting variety. preprint (2006)

[Sc-Ya] B. Schuster and N. Yagita, Transfers of Chern classes in BP-cohomology and Chow rings, Trans. Amer. Math. Soc. 353 (2001), no. 3, 1039-1054 (electronic).

[Su-Jo] A.Suslin and S.Joukhovitski. Norm Variety. J.Pure and Appl. Algebra 206 (2006) 245-276.

[Th] C. Thomas. Filtrations on the representation ring of a finite group. Contempt. math. 19 (1983), 397-405.

[Tod] H.Toda. On the cohomology ring of some homogeneous spaces. J. Math. Kyoto Univ. 15 (1975), 185-199.

[Tod-Wa] H.Toda and T.Watanabe. The integral cohomology ring of $F_{4} / T$ and $E_{6} / T$. J. Math. Kyoto Univ. 14 (1974), 257-286.

[To] B. Totaro. The Chow ring of classifying spaces. Proc.of Symposia in Pure Math. "Algebraic K-theory" (1997:University of Washington,Seattle) 67 (1999), 248-281.

[Vi-Ya] A.Vishik and N.Yagita. Algebraic cobordisms of a Pfister quadric. J. London Math. Soc. 76 (2007) 586-604.

[Vit] A. Vistoli. On the cohomology and the Chow ring of the classifying space $P G L_{p} . J$. Reine Angew. Math. 610 (2007), 181-227.

[Vo1] V. Voevodsky. The Milnor conjecture. www.math.uiuc.edu/K-theory/0170 (1996).

[Vo2] V. Voevodsky (Noted by C.Weibel). Voevodsky's Seattle lectures : $K$-theory and motivic cohomology Proc.of Symposia in Pure Math. "Algebraic K-theory" (1997:University of Washington, Seattle) 67 (1999), 283-303.

[Vo3] V. Voevodsky. Motivic cohomology with $\mathbb{Z} / 2$ coefficient. Publ. Math. IHES 98 (2003), 59-104.

[Vo4] V.Voevodsky. Reduced power operations in motivic cohomology. Publ.Math. IHES 98 (2003), $1-57$.

[Vo5] V.Voevodsky. On motivic cohomology with $\mathbb{Z} / l$-coefficients. Ann. Math. 174 (2011) 401-438.

[Wi] S. Wilson. The complex cobordism of $B O(n)$. J. London Math. Soc. 29 (1984), 352366. .

[Ya1] N. Yagita Chomology for groups of $\operatorname{rank}_{p} G=2$ and Brown-Peterson cohomology. $J$. Math. Soc. Japan 45 (1993), 627-644.

[Ya2] N. Yagita. Applications of Atiyah-Hirzebruch spectral sequence for motivic cobordism. Proc. London Math. Soc. 90 (2005) 783-816.

[Ya3] N. Yagita. Chow rings of nonabelian $p$-groups of order $p^{3}$. J. Math. Soc. Japan. 64 (2012) 507-531.

[Ya4] N. Yagita. Algebraic BP-theory and norm varieties. Hokkaido J. Math. J. 41 (2012), 275-316. .

[Ya5] N. Yagita. Note on Chow rings of nontrivial G-torsors over a field. Kodai Math. J. 34 (2011), 446-463. .

[Za] K. Zainoulline. Twisted gamma filtration of a linear algebraic group. Compositio math. 148 (2012), 1645-1654.

faculty of Education, Ibaraki University, Mito, Ibaraki, Japan

E-mail address: yagita@mx.ibaraki.ac.jp, 